\title{
Safety And Efficacy Of The Unique Opioid Buprenorphine For The Treatment Of Chronic Pain
}

Joseph V Pergolizzi Jr ${ }^{1,2}$

Robert B Raffa ${ }^{3,4}$

'NEMA Research, Inc., Naples, FL, USA;

${ }^{2}$ Neumentum, Palo Alto, CA, USA;

${ }^{3}$ University of Arizona College of

Pharmacy, Tucson, AZ, USA;

${ }^{4}$ Department of Pharmaceutical Sciences, Temple University School of Pharmacy,

Philadelphia, PA, USA
Correspondence: Joseph V Pergolizzi Jr NEMA Research, Inc., 868 106th Ave. North, Naples, FL 34108, USA

Tel + I 239-597-3564

$\mathrm{Fax}+$ I 239-908-4436

Email jpjmd@msn.com
This article was published in the following Dove Press journal: Journal of Pain Research

Background: Chronic pain is associated with decreased quality of life and is one of the most common reasons adults seek medical care, making treatment imperative for many aspects of patient well-being. Chronic pain management typically involves the use of Schedule II full $\mu$-opioid receptor agonists for pain relief; however, the increasing prevalence of opioid addiction is a national crisis that is impacting public health and social and economic welfare. Buprenorphine is a Schedule III partial $\mu$-opioid receptor agonist that is an equally effective but potentially safer treatment option for chronic pain than full $\mu$-opioid receptor agonists. The purpose of this review is to provide an overview of the clinical efficacy and safety of the transdermal and buccal formulations of buprenorphine, which are approved by the Food and Drug Administration for chronic pain, compared with that of extended-release full $\mu$-opioid receptor agonists.

Methods: Controlled or randomized controlled clinical trial information was retrieved from EMBASE, Medline, and PubMed using the search terms "buprenorphine" AND "chronic" AND "pain."

Results: A total of 33 clinical studies were ultimately used in this review, including 29 $(88 \%)$ on transdermal buprenorphine and $4(12 \%)$ on buprenorphine buccal film. Although the measure of pain intensity varied among studies, each of these 33 trials demonstrated efficacy for buprenorphine in pain relief. A total of 28 studies also assessed safety, with each concluding that buprenorphine was generally well tolerated.

Conclusion: Comparison of current clinical data along with results of responder and safety analyses support the use of buprenorphine over full $\mu$-opioid receptor agonists for effective preferential treatment of chronic pain; however, head-to-head clinical studies are warranted. Keywords: opioids, Schedule III, partial $\mu$-opioid receptor agonist

\section{Introduction}

Chronic pain is ongoing pain that persists for 6 months or more; it is one of the most common reasons adults seek medical care. ${ }^{1}$ It is estimated that 50 million adults in the United States experience chronic pain, and 19.6 million adults experience high-impact chronic pain, which frequently limits life or work activities. ${ }^{1}$ Chronic pain has been linked to various comorbidities including anxiety, depression, and suicide. ${ }^{1,2}$ Achieving adequate pain relief is therefore important for improving quality of life in patients with chronic pain.

Chronic pain treatment typically involves Schedule II full $\mu$-opioid receptor analgesics; however, the high and increasing prevalence of opioid addiction is a 
serious national crisis for public health and social and economic welfare. ${ }^{3,4}$ Opioid addiction creates challenges for the patient and physician in effectively treating chronic pain while adhering to state-mandated regulations and preventing misuse and addiction. ${ }^{5-7}$ The management of chronic pain with potentially safer yet equally effective treatment options is needed.

Buprenorphine is a relatively modern atypical opioid that is derived from the opium alkaloid thebaine of the poppy Papaver somniferum. ${ }^{8}{ }^{8}$ It has been used as an analgesic in the United States since $1981 .^{10,11}$ Buprenorphine functions by targeting the opioid receptors $\mu, \delta$, and $\kappa$ and opioid receptorlike 1 (ORL1). ${ }^{4}$ Multimechanistic effects are observed in vitro depending on the receptor subtype, as buprenorphine is a partial agonist at the $\mu$-opioid receptor, an antagonist at the $\delta$ - and $\kappa$-opioid receptors, and an agonist at ORL1. ${ }^{9}$

Because the $\mu$-opioid receptor is well-known for its role in analgesia, misconceived notions regarding the efficacy of buprenorphine as an analgesic have been based on its classification as a partial agonist; ${ }^{12-14}$ however, partial agonism at the $\mu$-opioid receptor does not limit the full analgesic efficacy of buprenorphine and in fact may explain the decreased likelihood of respiratory depression and abuse potential. ${ }^{13,15-18}$ In addition, the antagonistic effects of buprenorphine at the $\delta$ - and $\kappa$-opioid receptors may contribute to its favorable safety and tolerability profile by decreasing the risks of respiratory depression, constipation, and suicidal tendencies, as well as potentiating anti-depressant and anti-anxiety effects. ${ }^{9,19-22}$ Agonistic activity at ORL1 may also contribute to buprenorphine's analgesic efficacy, as it has been shown to promote analgesia in the dorsal horn of the spinal cord. ${ }^{23}$

Treatment options for chronic pain, such as buprenorphine, are especially important when the United States is facing increasing opioid misuse and related overdoses. ${ }^{24,25}$ Because buprenorphine has less potential for abuse than drugs or substances in Schedules I and II, it has been classified by the Drug Enforcement Administration (DEA) as a Schedule III controlled substance. ${ }^{21}$ Orally administered buprenorphine has only approximately $10 \%$ bioavailability, but recent advances in drug delivery circumvent this issue. ${ }^{26}$ The sublingual formulation provides approximately $28-51 \%$ bioavailability, the transdermal formulation provides approximately $15 \%$, and the buccal film exhibits $46-65 \%$ bioavailability. Each of these formulations bypasses first-pass metabolism. ${ }^{24,27-30}$

Buprenorphine transdermal system (Butrans ${ }^{\circledR}$, Purdue Pharma, LP, Stamford, CT) and buprenorphine buccal film
(Belbuca ${ }^{\circledR}$, BioDelivery Sciences International, Inc, Raleigh, NC) are the two formulations currently indicated for the management of pain that is severe enough to require daily, around-the-clock, long-term opioid treatment and for which alternative treatment options are inadequate. ${ }^{23,24}$ This standard labeling is required on all extended-release (ER) or long-acting opioids indicated for chronic pain. The purpose of this review is to provide an overview of the clinical efficacy and safety of these Food and Drug Administration (FDA)-approved buprenorphine formulations and to elaborate on the current data that support its effective and potentially safer use in chronic pain management compared with ER Schedule II opioids.

\section{Materials And Methods}

EMBASE, Medline, and PubMed searches were conducted on April 11, 2019, using the terms "buprenorphine" AND "chronic" AND "pain". The search was restricted to controlled or randomized controlled clinical trials in humans that were published in English and for which full texts were available (ie, not a meeting abstract). Articles that did not include standard outcomes of pain intensity or quality of life measures were considered irrelevant and were not included. Other references were added at the authors' discretion. A reduction in pain or improvement in quality of life that was considered by the authors of the study to provide utility for transdermal buprenorphine or buprenorphine buccal film in the management of chronic pain was regarded as a positive overall outcome.

\section{Results}

A total of 118 studies were assessed for eligibility. The abstracts were screened and analyzed, resulting in the exclusion of 85 studies. A total of 33 clinical studies obtained with the search criteria were ultimately included in this review, 29 (88\%) of which examined transdermal buprenorphine and $4(12 \%)$ of which examined buprenorphine buccal film (Figure 1).

\section{Transdermal Formulation}

The safety and efficacy of transdermal buprenorphine has been studied in multiple chronic pain populations (Table 1). ${ }^{31-59}$ On the basis of the search criteria used here, $12(41 \%)$ studies examined general chronic pain, $10(34 \%)$ examined chronic low back pain, 5 (17\%) examined osteoarthritis pain, 1 (3\%) examined chronic malignant pain, and $1(3 \%)$ examined musculoskeletal pain. The duration of these studies ranged from 6 days to 5.7 years, 


\section{Identification}

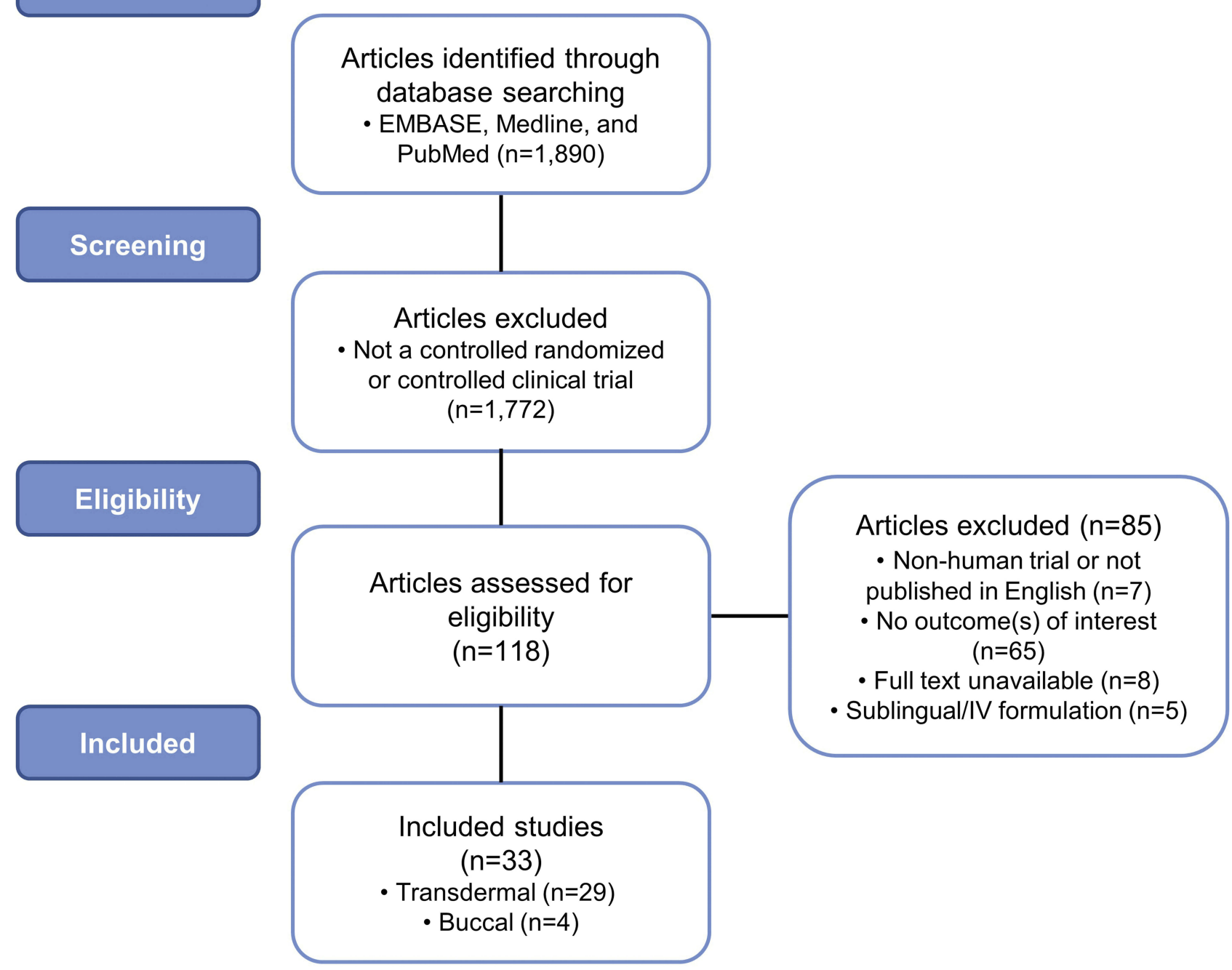

Figure I Flow diagram: clinical trial identification and inclusion. Schematic detailing the search criteria used in this review to identify relevant clinical trials of buprenorphine in chronic pain management.

with the doses of transdermal buprenorphine ranging from 5 to $140 \mu \mathrm{g} / \mathrm{h}$ (in the United States, $20 \mu \mathrm{g} / \mathrm{h}$ is the highest dosage strength available). A total of 11 (38\%) studies were placebo controlled, 7 (24\%) had no comparator, 7 (24\%) compared against buprenorphine dose, duration, or formulation differences, $3(10 \%)$ used an analgesic comparator, 2 (7\%) used age comparators, and 1 (3\%) compared against supplemental analgesics. Examination of multiple comparators by some studies was taken into account.

All 12 studies of general chronic pain demonstrated the efficacy of transdermal buprenorphine in pain relief, including $2(17 \%)$ studies assessing an elderly population. ${ }^{31,33,36,40-42,48-52,55}$ Of these, 4 (33\%) studies also observed various quality of life parameters, with all
4 showing improvements in general quality of life, sleep duration, and/or need for breakthrough analgesia. ${ }^{31,33,50,52}$ All 12 of these studies also found transdermal buprenorphine to be well tolerated in the treatment of general chronic pain. ${ }^{31,33,36,40-42,48-52,55}$

All of the 15 studies examining chronic low back pain (10 studies) or osteoarthritis pain (5 studies) demonstrated effective pain relief for transdermal buprenorphine, including 1 osteoarthritis study that examined solely elderly populations. ${ }^{14,34,35,37,38,43,44,46,47,53,54,56-59}$ These studies also examined parameters associated with quality of life, and all 15 demonstrated improvements in activities of daily living (lifting, bending, working), sleep, and/or physical and mental health in response to treatment with transdermal buprenorphine. . $^{14,34,35,37,38,43,44,46,47,53,54,56-59}$ Safety was 


\begin{tabular}{|c|c|c|c|c|c|}
\hline 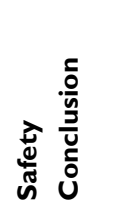 & 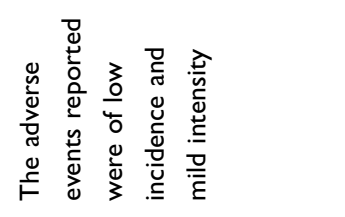 & 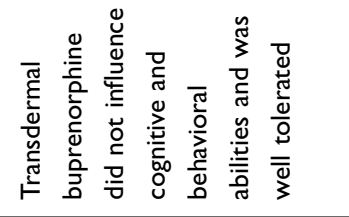 & 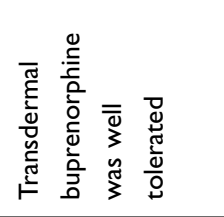 & 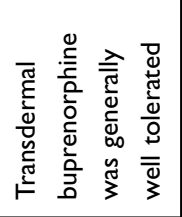 & 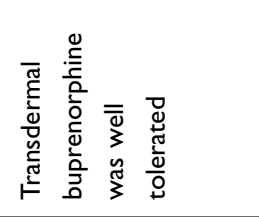 \\
\hline 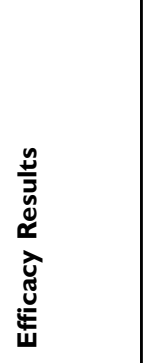 & 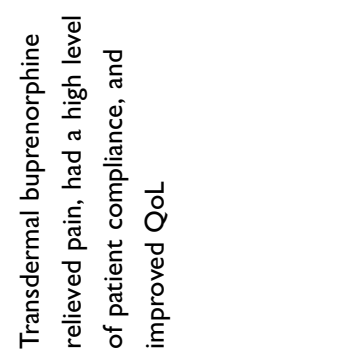 & 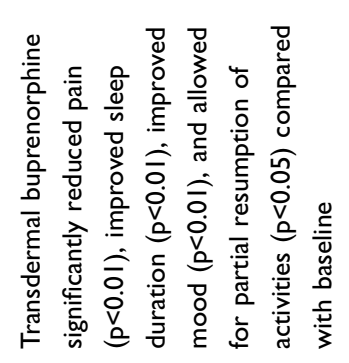 & 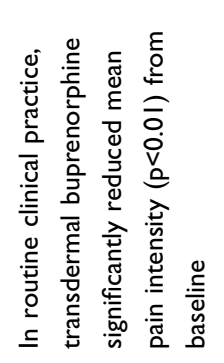 & 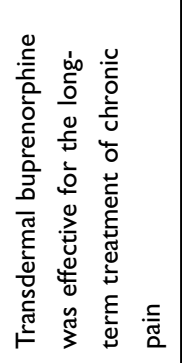 & 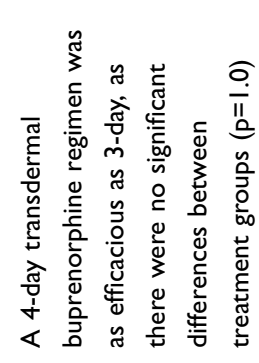 \\
\hline 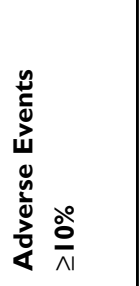 & 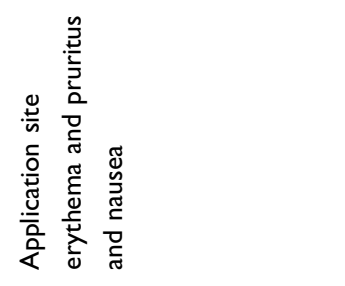 & 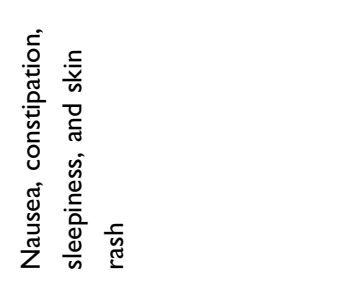 & $\begin{array}{l}0 \\
\stackrel{0}{0} \\
z\end{array}$ & 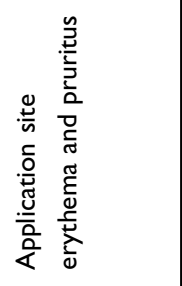 & 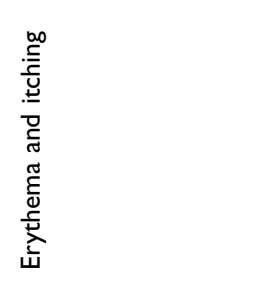 \\
\hline 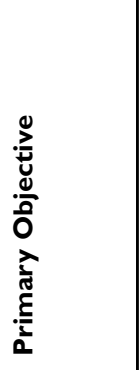 & 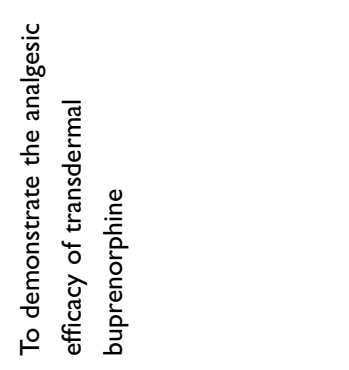 & 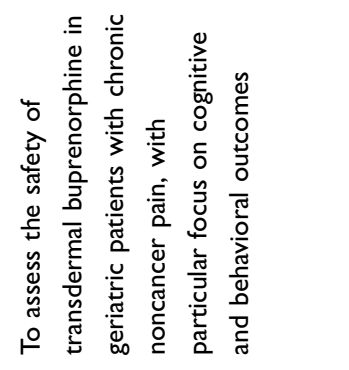 & 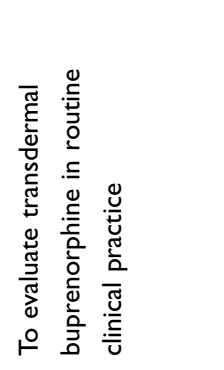 & 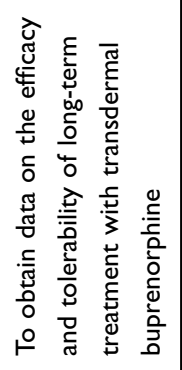 & 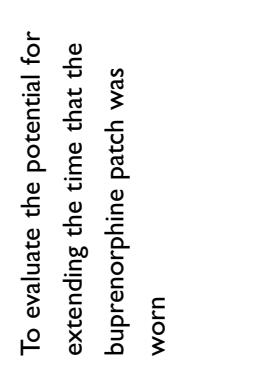 \\
\hline 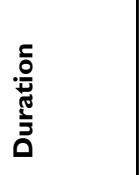 & 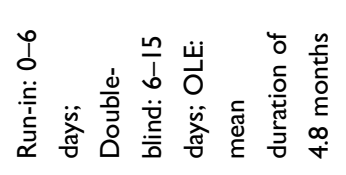 & 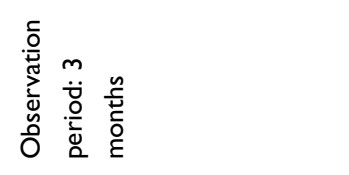 & 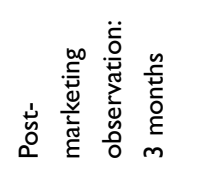 & 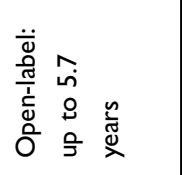 & 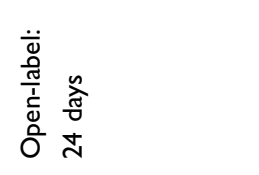 \\
\hline 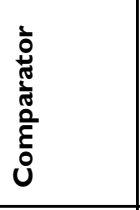 & $\begin{array}{l}0 \\
\stackrel{8}{\mathbb{U}} \\
\frac{\pi}{\alpha} \\
\end{array}$ & 1 & 1 & 1 & 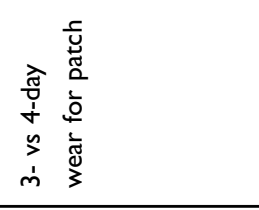 \\
\hline 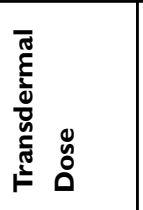 & 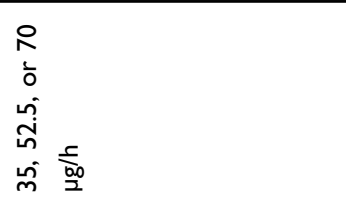 & 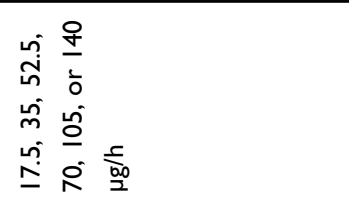 & 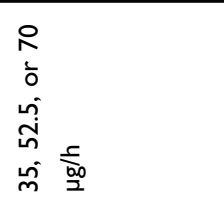 & 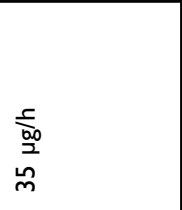 & 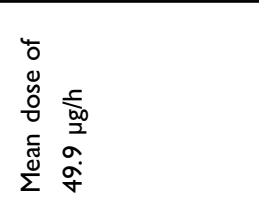 \\
\hline$\frac{0}{\frac{0}{0}}$ & 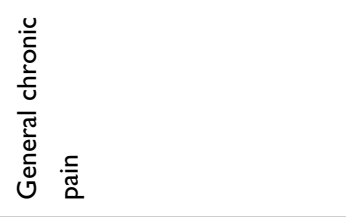 & 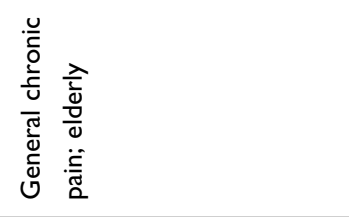 & 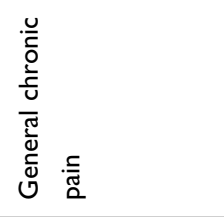 & 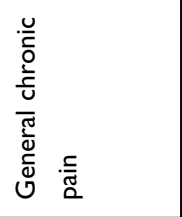 & 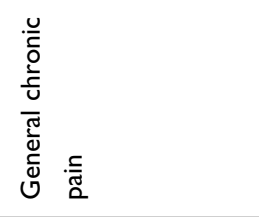 \\
\hline 蒙总 & 紊 & 宽 & 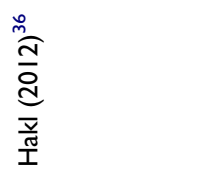 & 咅亮 & 雍 \\
\hline
\end{tabular}




\begin{tabular}{|c|c|c|c|}
\hline 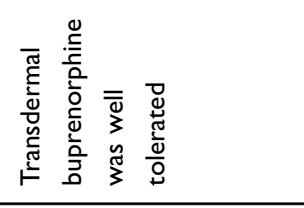 & 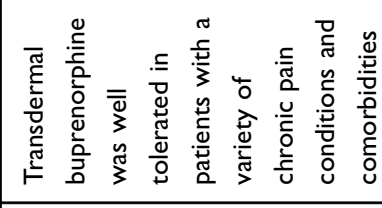 & 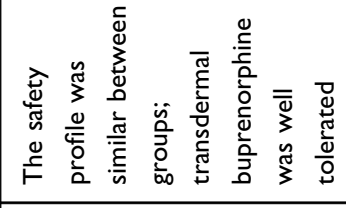 & 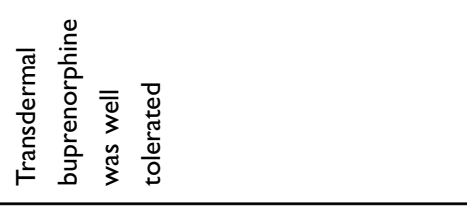 \\
\hline 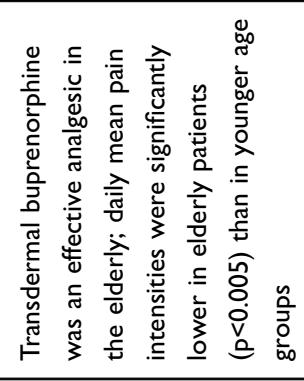 & 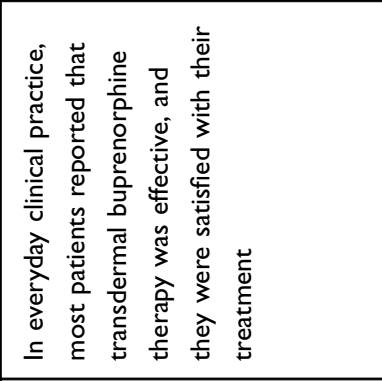 & 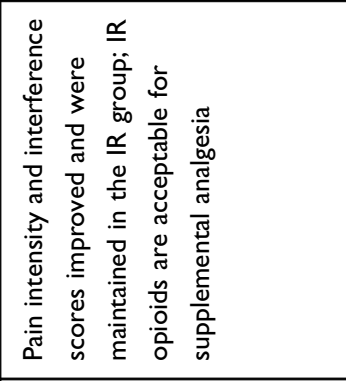 & 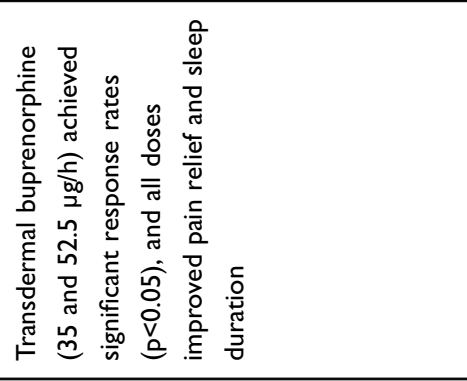 \\
\hline 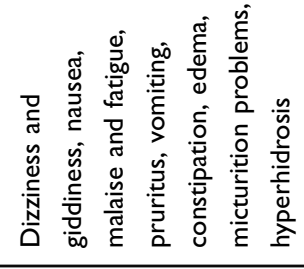 & 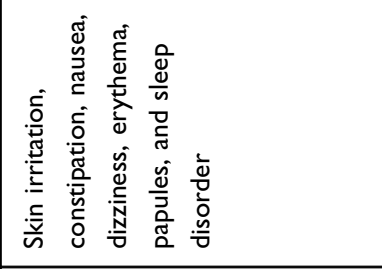 & 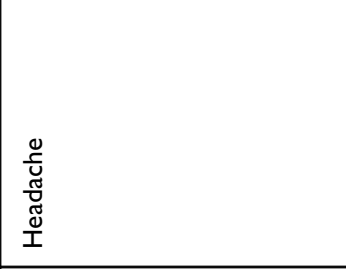 & 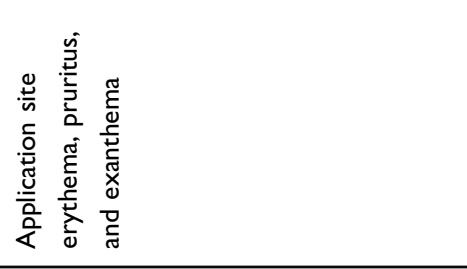 \\
\hline 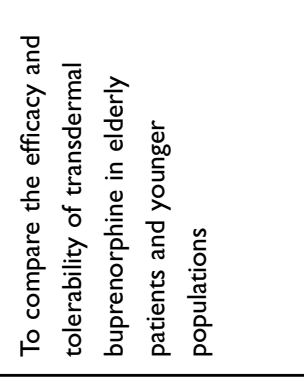 & 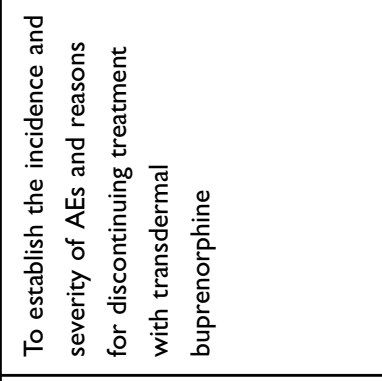 & 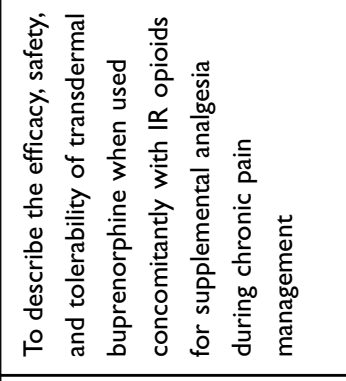 & 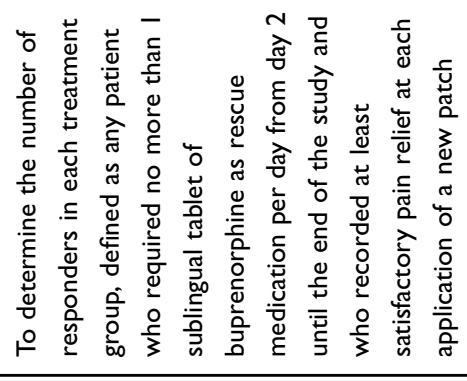 \\
\hline 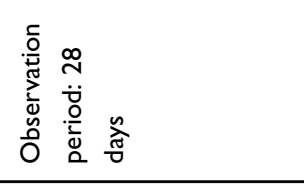 & 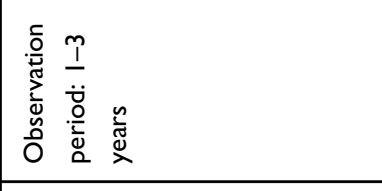 & 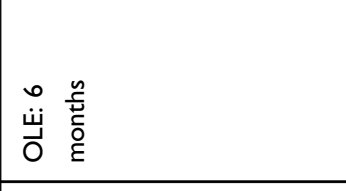 & 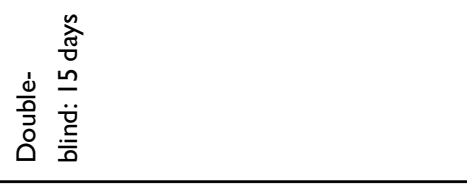 \\
\hline 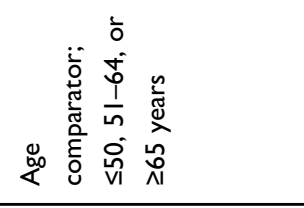 & 1 & 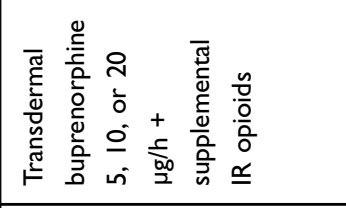 & 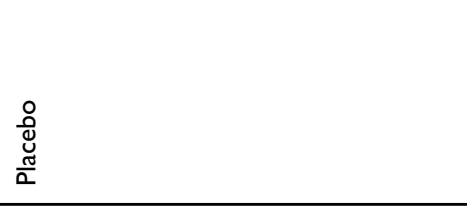 \\
\hline 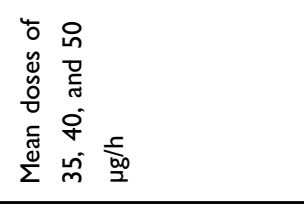 & 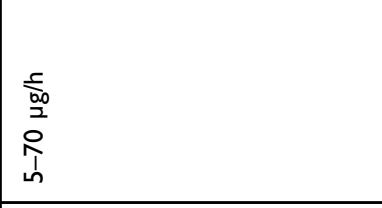 & 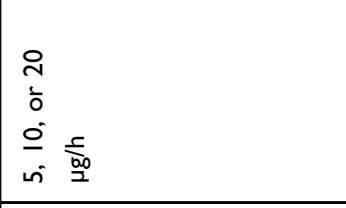 & 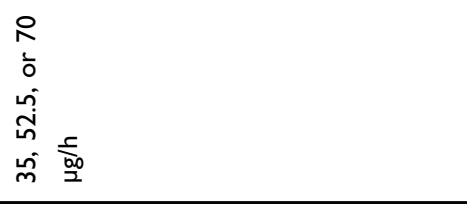 \\
\hline 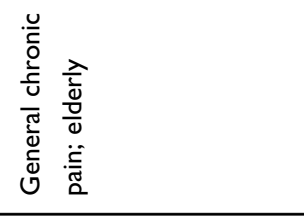 & 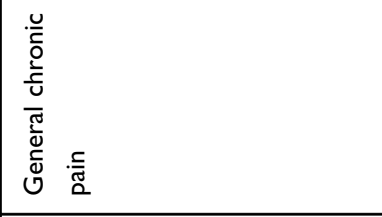 & 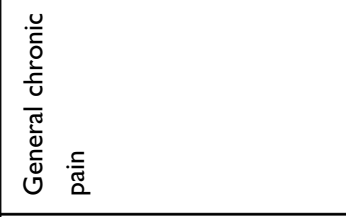 & 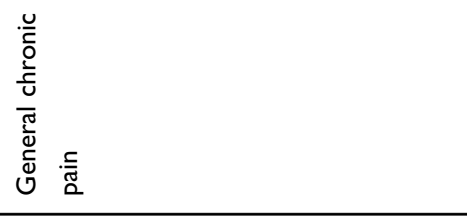 \\
\hline 蒿 & 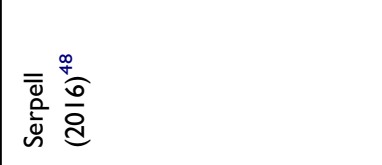 & 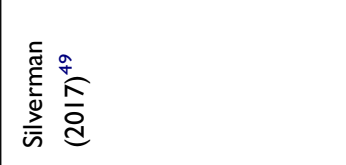 & 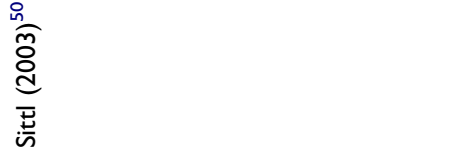 \\
\hline
\end{tabular}




\begin{tabular}{|c|c|c|c|c|}
\hline 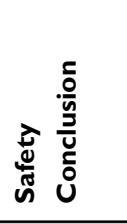 & 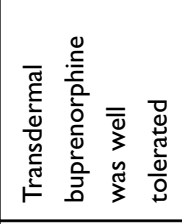 & 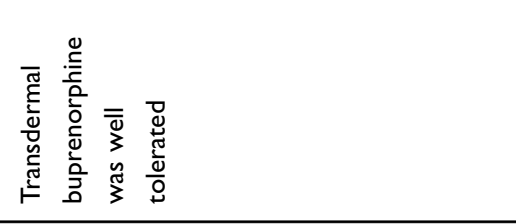 & 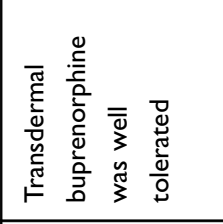 & 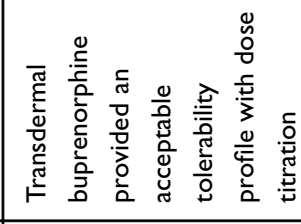 \\
\hline 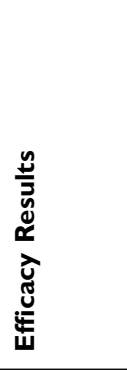 & 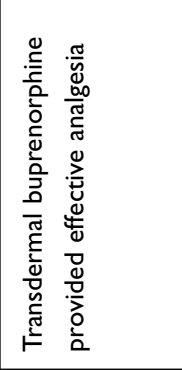 & 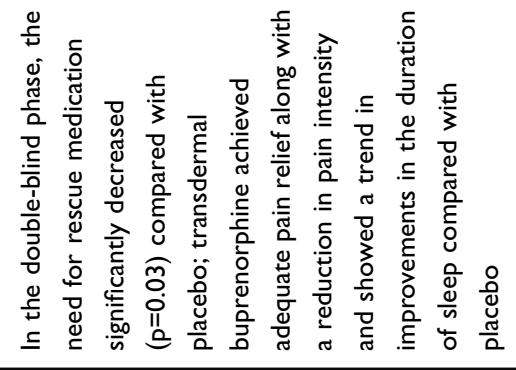 & 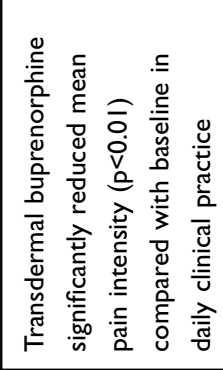 & 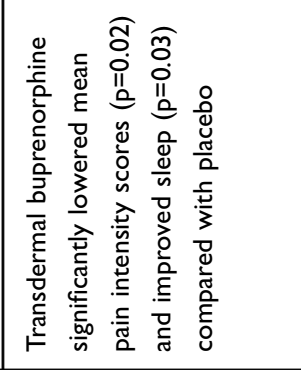 \\
\hline 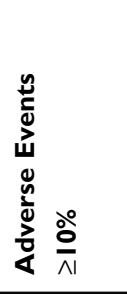 & 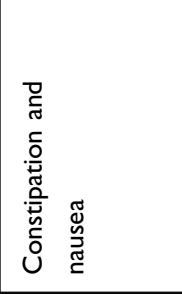 & 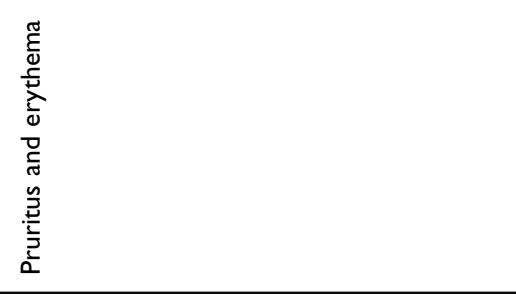 & $\begin{array}{l}0 \\
\text { o } \\
z\end{array}$ & 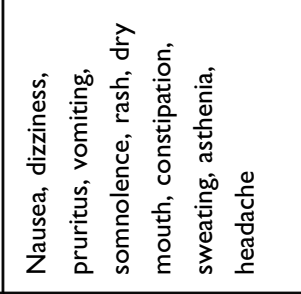 \\
\hline 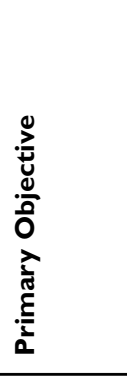 & 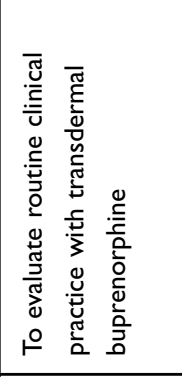 & 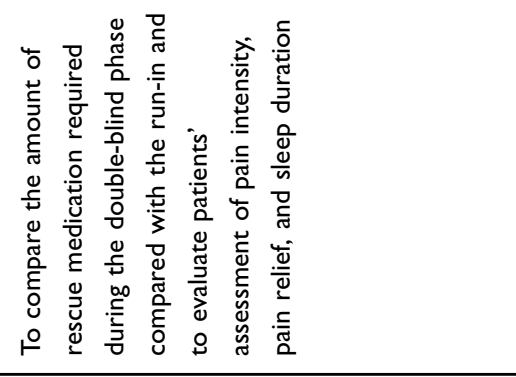 & 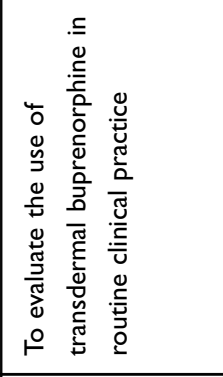 & 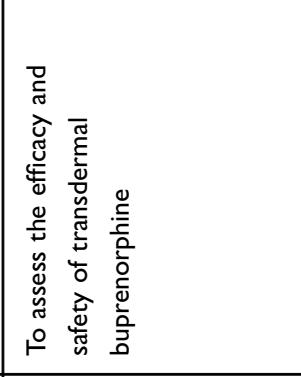 \\
\hline 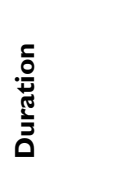 & 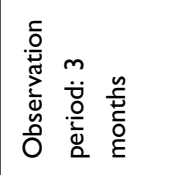 & 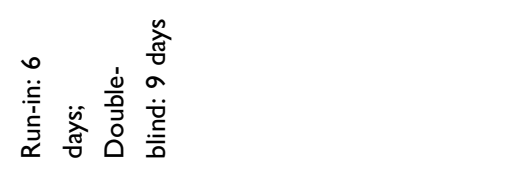 & 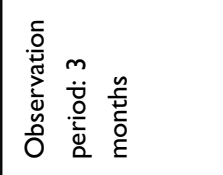 & 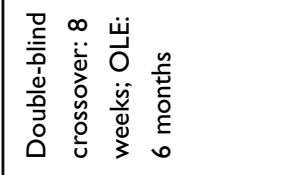 \\
\hline 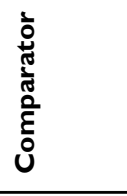 & 1 & $\begin{array}{l}\circ \\
\stackrel{8}{\mathbb{d}} \\
\frac{\tilde{w}}{0} \\
\end{array}$ & 1 & $\begin{array}{l}\circ \\
\stackrel{8}{0} \\
\frac{\tilde{\sigma}}{\alpha} \\
\frac{\sigma}{\alpha}\end{array}$ \\
\hline 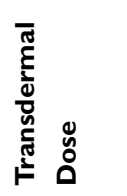 & 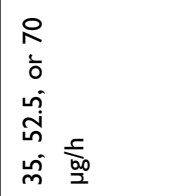 & 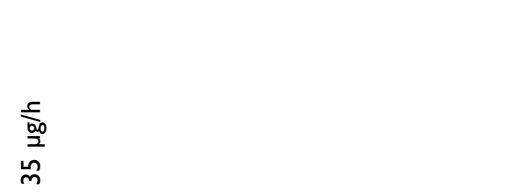 & 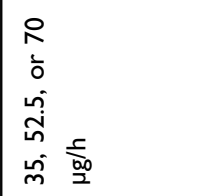 & $\begin{array}{l}\frac{1}{20} \\
\frac{\text { bo }}{2} \\
0 \\
0 \\
0\end{array}$ \\
\hline $\begin{array}{l}\frac{5}{0} \\
\frac{\pi}{3} \\
\frac{\pi}{0} \\
\frac{0}{2}\end{array}$ & 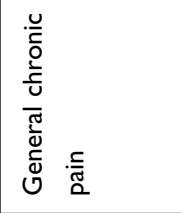 & 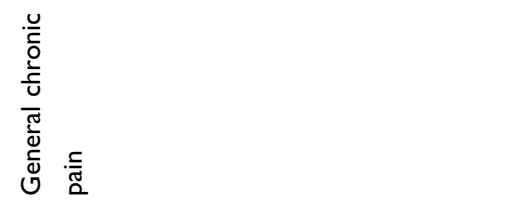 & 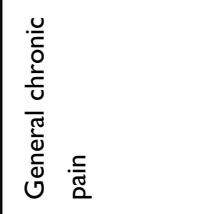 & 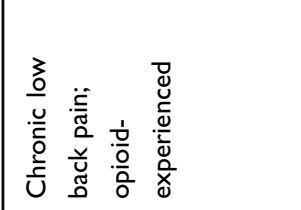 \\
\hline 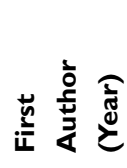 & 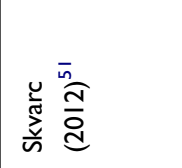 & 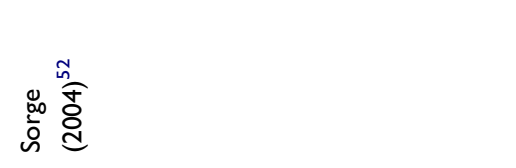 & 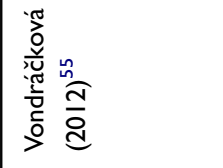 & 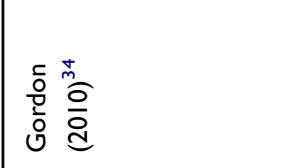 \\
\hline
\end{tabular}




\begin{tabular}{|c|c|c|c|}
\hline 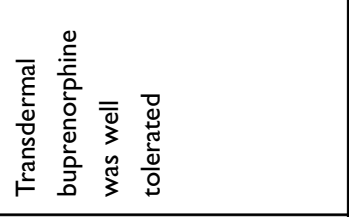 & 1 & 1 & 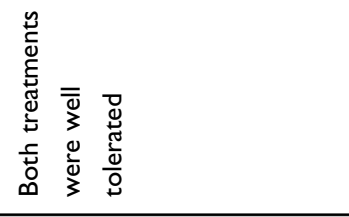 \\
\hline 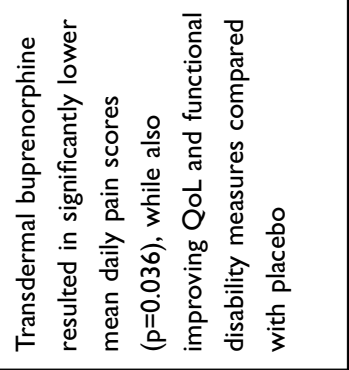 & 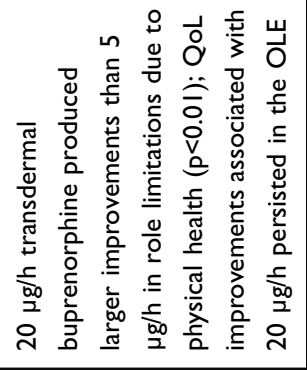 & 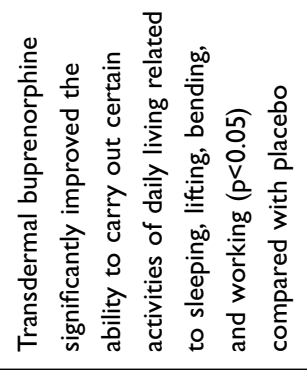 & 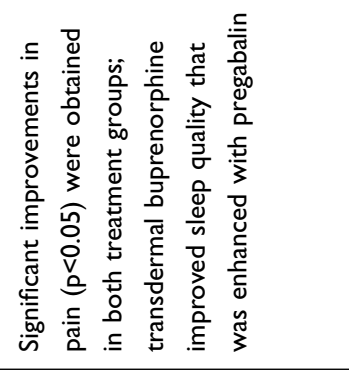 \\
\hline 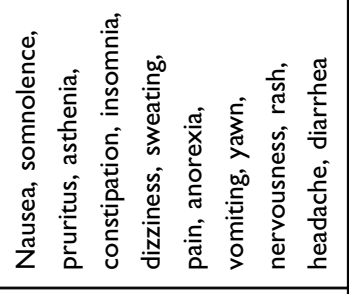 & 1 & 1 & 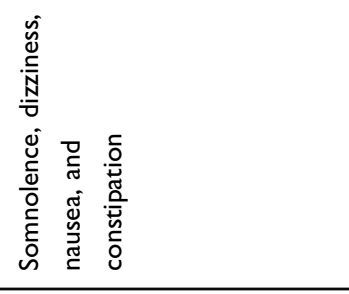 \\
\hline 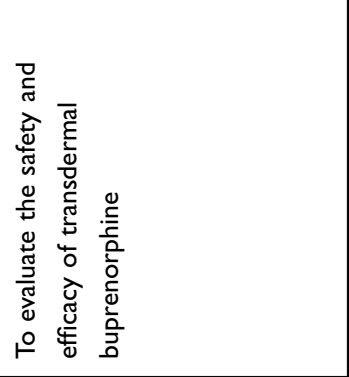 & 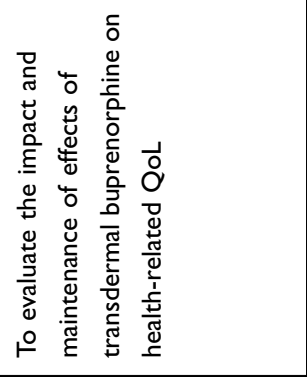 & 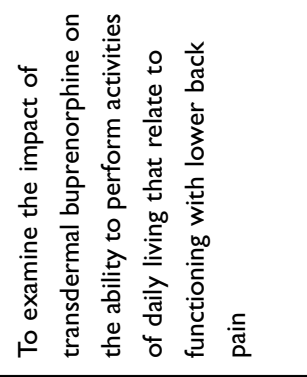 & 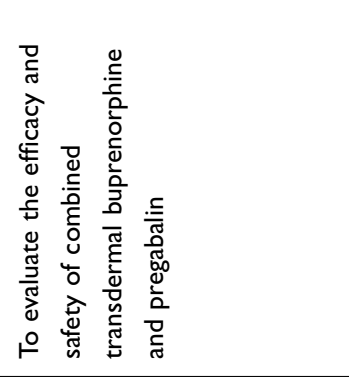 \\
\hline 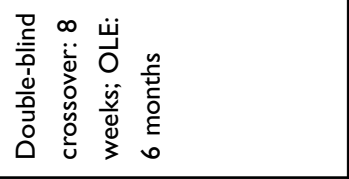 & 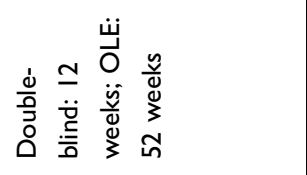 & 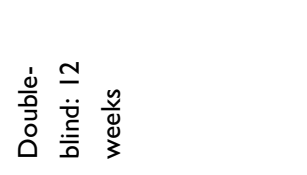 & 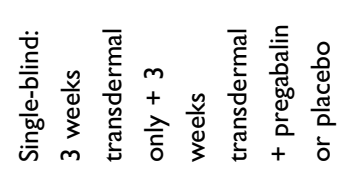 \\
\hline $\begin{array}{l}\circ \\
\frac{8}{4} \\
\frac{\pi}{\alpha} \\
\frac{\pi}{\alpha} \\
\end{array}$ & 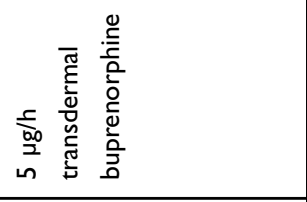 & $\begin{array}{l}\circ \\
\frac{8}{4} \\
\frac{\pi}{0} \\
\end{array}$ & 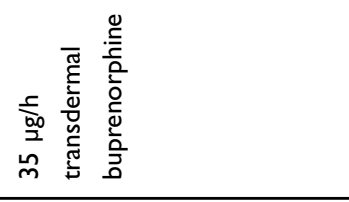 \\
\hline 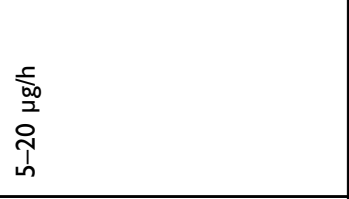 & $\begin{array}{l}\frac{c}{0.0} \\
\frac{0}{20} \\
\stackrel{i}{2}\end{array}$ & 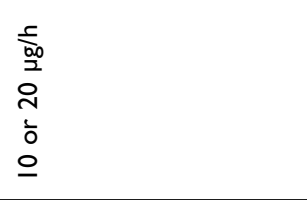 & 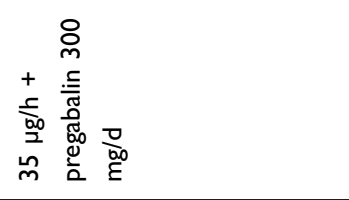 \\
\hline 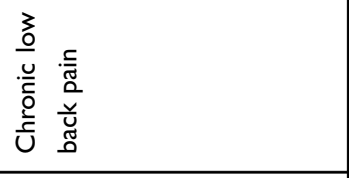 & 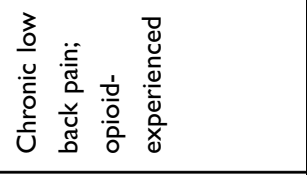 & 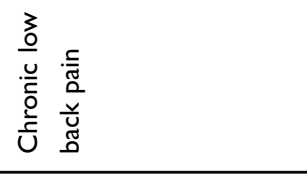 & 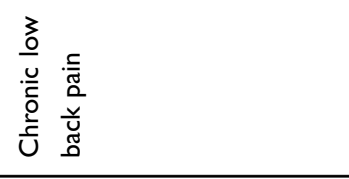 \\
\hline 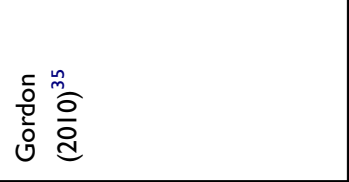 & 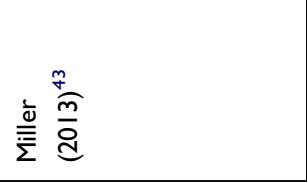 & 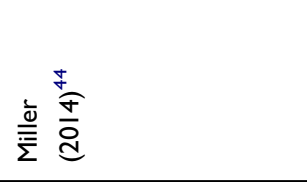 & 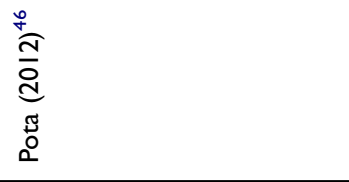 \\
\hline
\end{tabular}




\begin{tabular}{|c|c|c|c|c|}
\hline 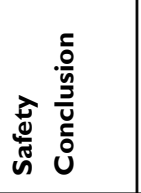 & 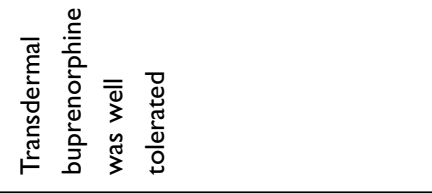 & 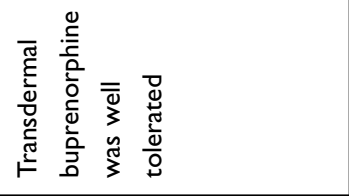 & 1 & 1 \\
\hline 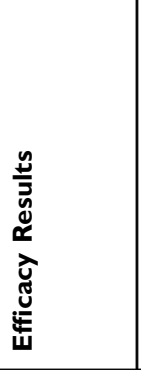 & 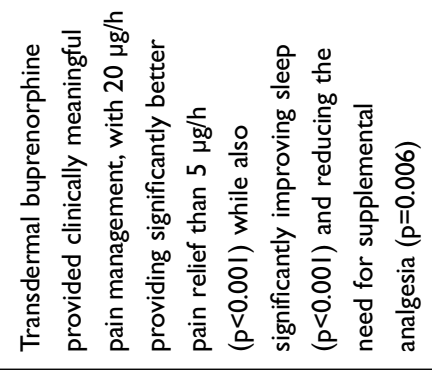 & 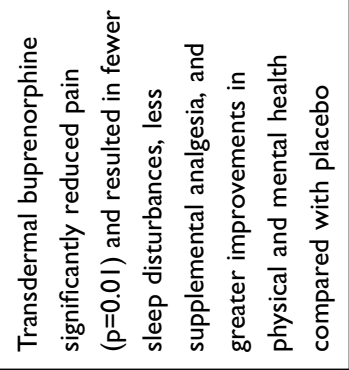 & 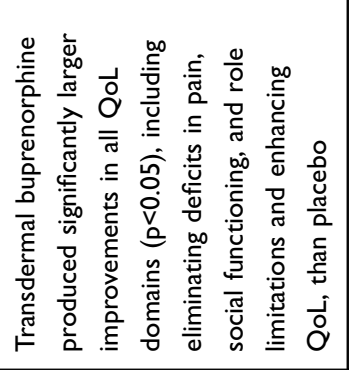 & 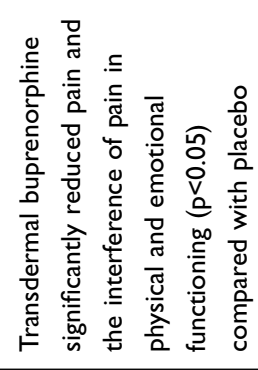 \\
\hline 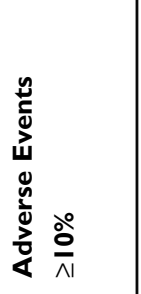 & 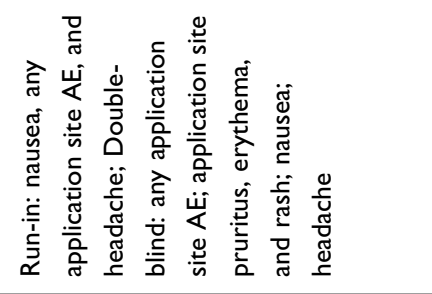 & 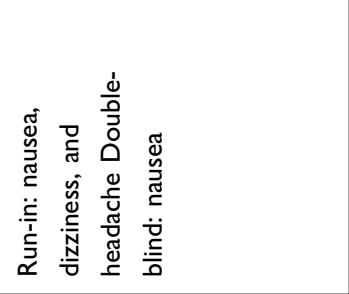 & I & 1 \\
\hline 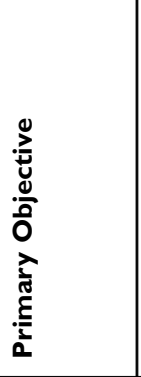 & 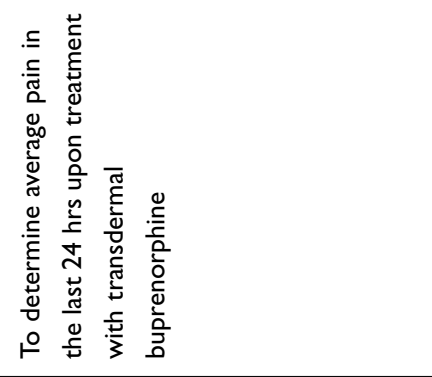 & 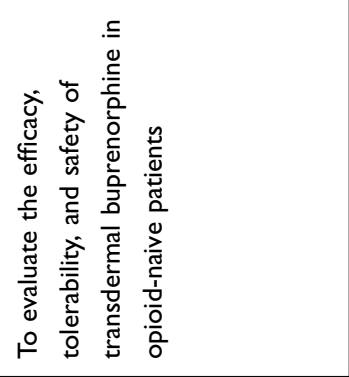 & 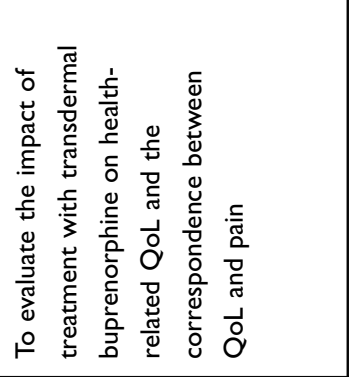 & 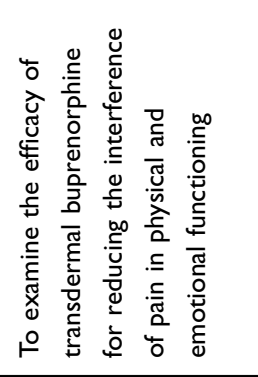 \\
\hline مَ & 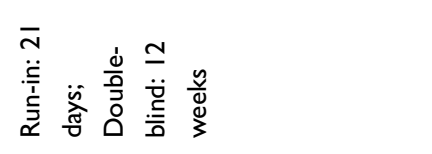 & 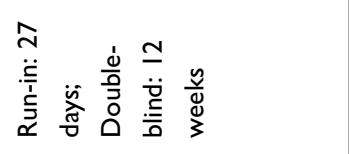 & 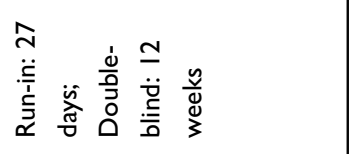 & 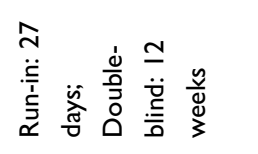 \\
\hline 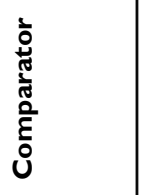 & 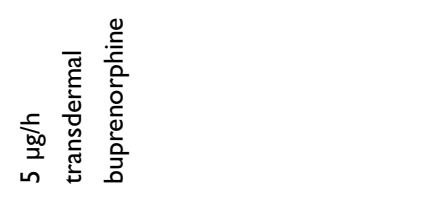 & $\begin{array}{l}\circ \\
\stackrel{8}{u} \\
\frac{\widetilde{\pi}}{\alpha}\end{array}$ & $\begin{array}{l}\circ \\
\stackrel{8}{u} \\
\frac{\tilde{g}}{0}\end{array}$ & 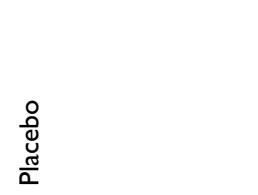 \\
\hline 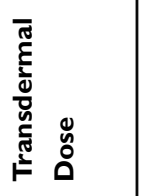 & $\begin{array}{l}\frac{5}{000} \\
\frac{\text { on }}{2}\end{array}$ & 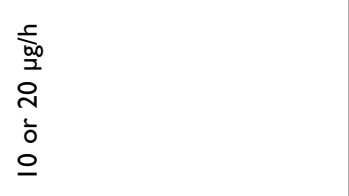 & $\begin{array}{l}\frac{1}{c} \\
\text { op } \\
\stackrel{1}{2} \\
\vdots \\
\vdots \\
0\end{array}$ & $\begin{array}{l}\frac{c}{50} \\
\text { bo } \\
\stackrel{2}{2} \\
\vdots \\
\vdots \\
0\end{array}$ \\
\hline$\frac{5}{\frac{0}{20}}$ & 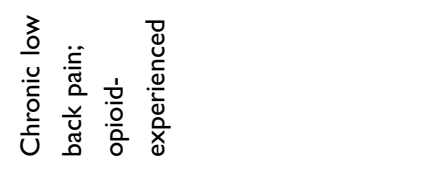 & 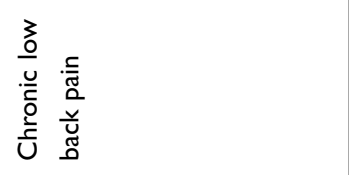 & 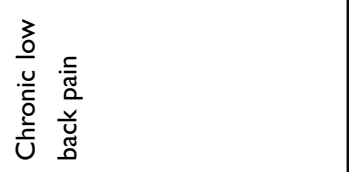 & 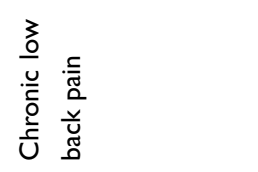 \\
\hline 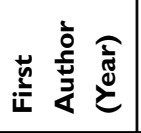 & 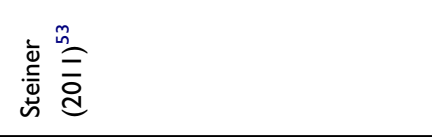 & 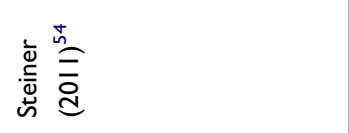 & 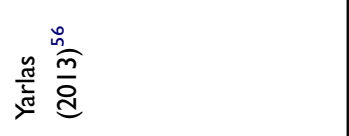 & $\frac{\frac{n}{2}}{\frac{\pi}{\pi}} \frac{\sqrt[n]{n}}{\frac{n}{d}}$ \\
\hline
\end{tabular}




\begin{tabular}{|c|c|c|c|c|}
\hline 1 & 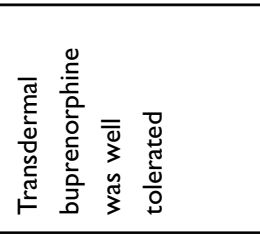 & 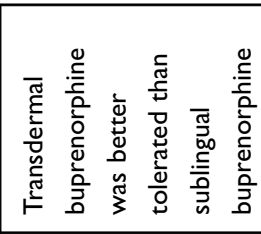 & 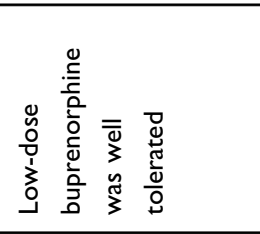 & 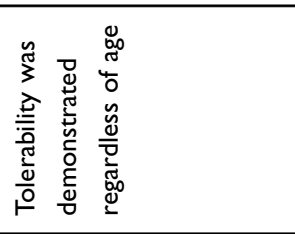 \\
\hline 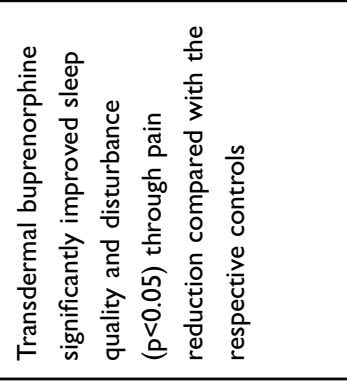 & 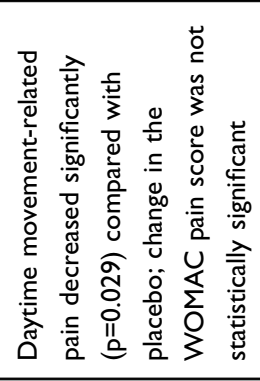 & 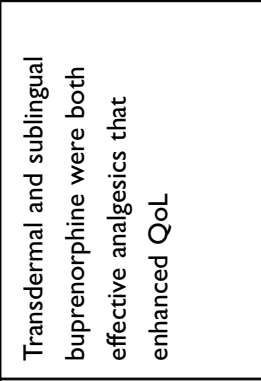 & 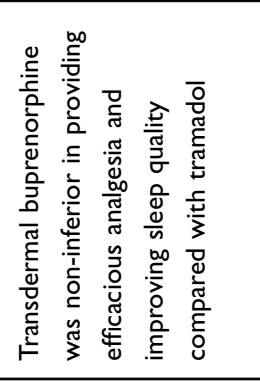 & 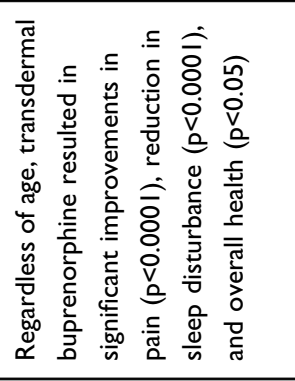 \\
\hline 1 & 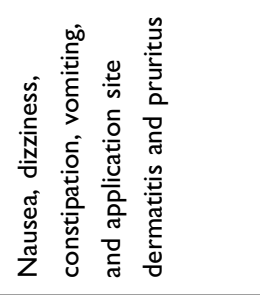 & 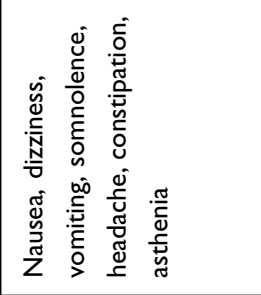 & 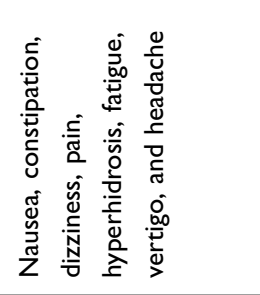 & 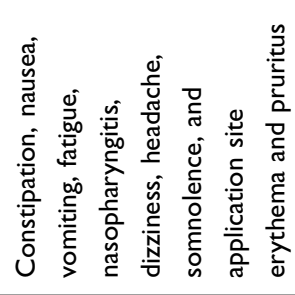 \\
\hline 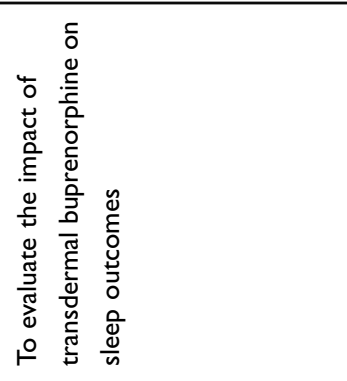 & 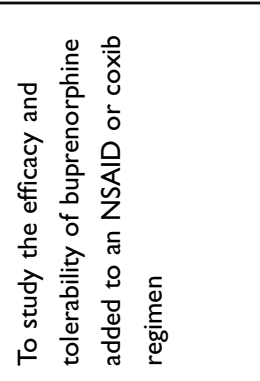 & 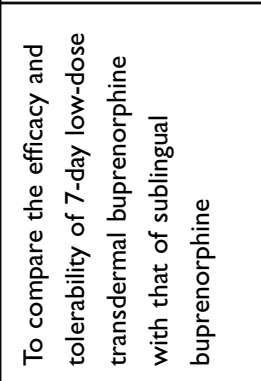 & 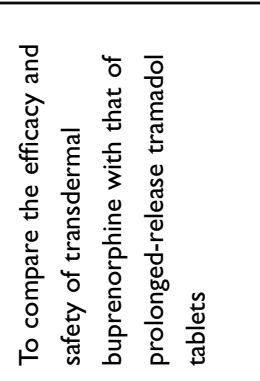 & 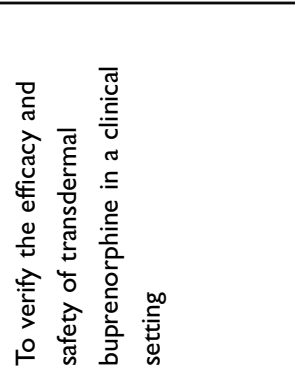 \\
\hline 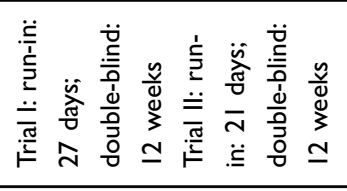 & 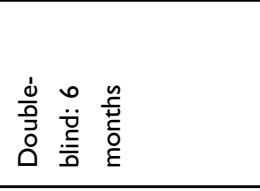 & 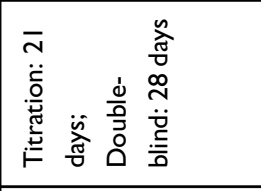 & 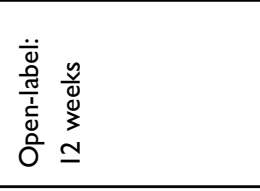 & 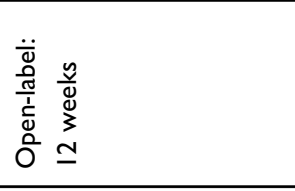 \\
\hline 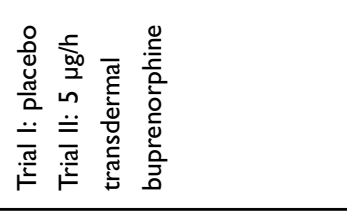 & 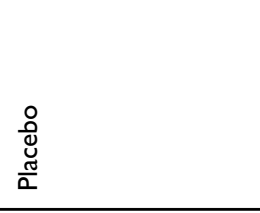 & 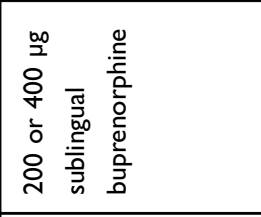 & 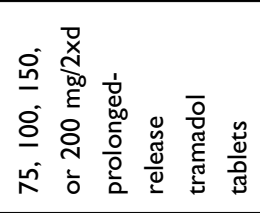 & 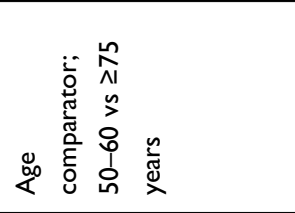 \\
\hline 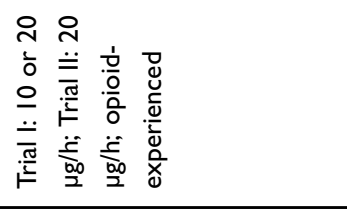 & 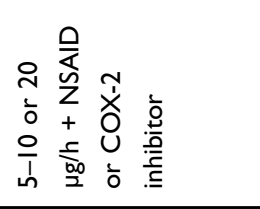 & 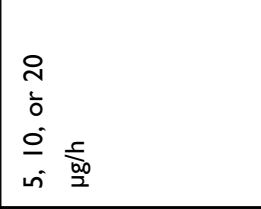 & 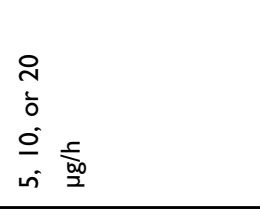 & 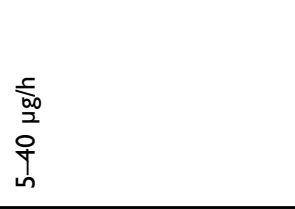 \\
\hline 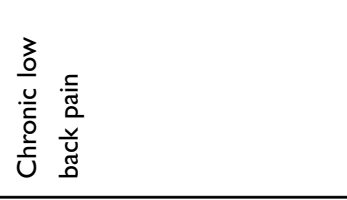 & 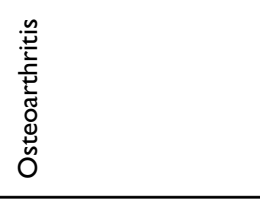 & 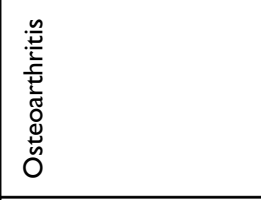 & 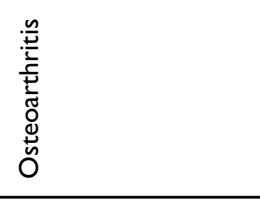 & 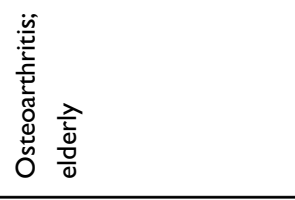 \\
\hline 竞 & 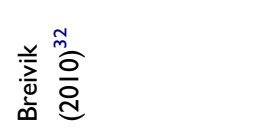 & 类 & 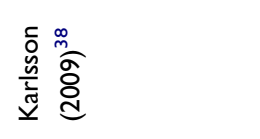 & 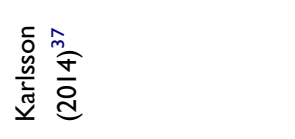 \\
\hline
\end{tabular}




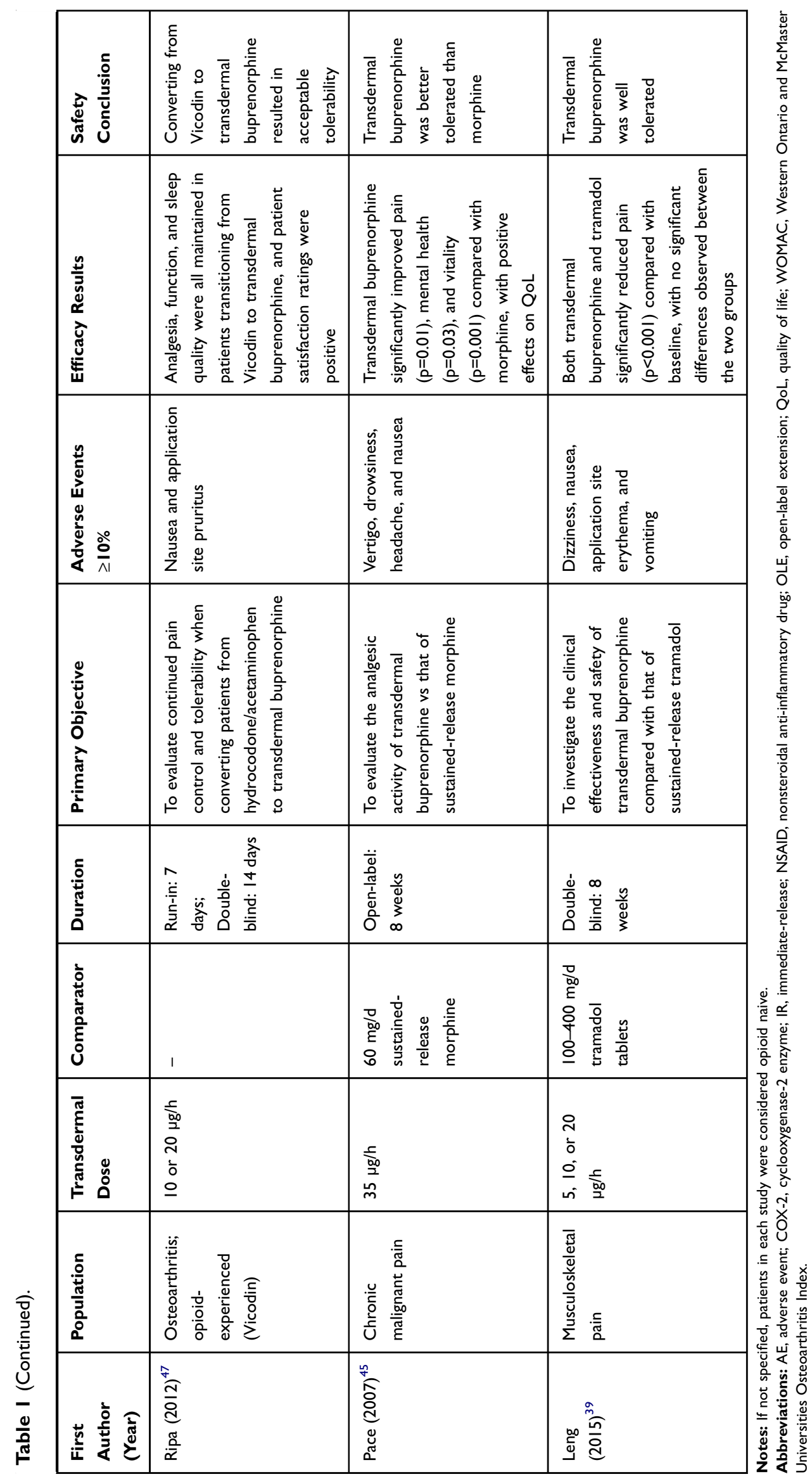


also examined in $5(50 \%)$ chronic low back pain studies and in each osteoarthritis study, and buprenorphine was generally well tolerated in patients with chronic low back and osteoarthritis pain. ${ }^{14,34,35,37,38,46,47,53,54,58}$

Similarly, the single study that examined chronic malignant pain found transdermal buprenorphine was well tolerated, improved pain relief, and enhanced mental health and vitality, with overall positive effects on quality of life. ${ }^{45}$ The study of transdermal buprenorphine on musculoskeletal pain observed pain reduction and also concluded that transdermal buprenorphine was well tolerated. ${ }^{39}$

Regarding the 3 studies that utilized analgesic comparators, 1 compared the safety and efficacy of buprenorphine with that of morphine and 2 with tramadol. In patients with chronic malignant pain, transdermal buprenorphine significantly improved pain $(\mathrm{p}=0.01)$, mental health $(\mathrm{p}=0.03)$, and vitality $(\mathrm{p}=0.001)$ compared with morphine and was better tolerated for chronic pain management. ${ }^{45}$ In patients with chronic musculoskeletal or osteoarthritis pain, buprenorphine was statistically noninferior to sustained-release tramadol, with the incidence of adverse events being comparable between the two groups. ${ }^{38,39}$

Although the measure of pain intensity varied among these studies depending on the type of chronic pain being assessed, in each case, the authors proposed utility for transdermal buprenorphine in maintaining, reducing, or providing relief from pain and/or enhancing the quality of life of patients with chronic pain. ${ }^{31-57}$ In each of the 24 studies that also assessed safety, transdermal buprenorphine was considered well tolerated. ${ }^{31-42,45-55,58}$ The most commonly reported adverse events in transdermal buprenorphine clinical trials were nausea, headache, application site pruritus, dizziness, constipation, somnolence, vomiting, application site erythema, dry mouth, and application site rash. ${ }^{23}$

\section{Buccal Formulation}

The safety and efficacy of buprenorphine buccal film has been studied in opioid-experienced and opioid-naive patients with chronic low back pain and in patients with general chronic pain (Table 2). ${ }^{26,60-62}$ Four clinical studies were identified with the search criteria used here; 1 was a 7-day crossover study, 2 were 12 -week double-blind studies, and 1 was a 48-week long-term safety study. The doses of buprenorphine buccal film ranged from 75 to 900 $\mu \mathrm{g} / 12 \mathrm{~h} .^{60-62}$

All four studies found that buprenorphine buccal film relieved pain or maintained pain relief. ${ }^{26,60-62}$ Nausea, constipation, headache, vomiting, fatigue, dizziness, somnolence, diarrhea, dry mouth, and upper respiratory tract infection were the most common adverse reactions reported in clinical trials, and buprenorphine buccal film was deemed generally well tolerated in each study. ${ }^{26,60-62}$ In addition, patient compliance in these studies was high, as indicated by the high number of completers and subsequent continuation in the long-term safety study. ${ }^{26,60-62}$

\section{Discussion}

\section{The Clinical Efficacy Of Buprenorphine In Chronic Pain}

Of the buprenorphine formulations currently approved by the FDA for the management of chronic pain, the transdermal formulation has been the most extensively studied, likely because of its indication and length of time on the market. ${ }^{23}$ The ability of transdermal buprenorphine to provide effective pain relief has been demonstrated in a variety of clinical studies assessing an array of chronic pain types, and patient compliance tends to be high because of ease of use. ${ }^{31-57,63}$ Three of the transdermal buprenorphine trials assessed here utilized opioid comparators, and the results of these studies indicated superiority to morphine in relieving chronic malignant pain or noninferiority to tramadol for osteoarthritis or musculoskeletal pain. ${ }^{38,39,45}$ In addition, a phase IV realworld clinical trial demonstrated that the analgesic efficacy of transdermal buprenorphine in patients with chronic malignant pain was comparable to that of the Schedule II opioids morphine, oxycodone, and fentanyl. ${ }^{64}$ In a meta-analysis of clinical trials, transdermal buprenorphine was also found to provide pain relief similar to that of transdermal fentanyl. ${ }^{65}$ Transdermal buprenorphine has thus been clinically shown to be effective in managing chronic pain in a manner similar to that of the Schedule II opioids morphine, oxycodone, and fentanyl.

Buprenorphine buccal film is a relatively new formulation, and as a result, few clinical studies have been published. ${ }^{24}$ This formulation showed analgesic efficacy in all of the currently available studies, and the efficacy data in opioid-naive patients are comparable with those observed in studies of the Schedule II opioid oxymorphone; ${ }^{61,66}$ however, a head-to-head study is needed for direct comparison. A high level of patient compliance has been observed with buprenorphine buccal film, as indicated by the high percentages of completers and those who continued in a long-term safety study. ${ }^{26,60,61}$ Current clinical data also 


\begin{tabular}{|c|c|c|c|c|}
\hline 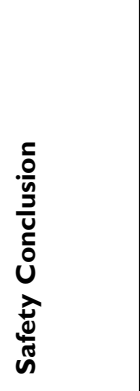 & 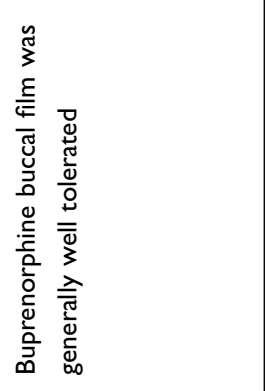 & 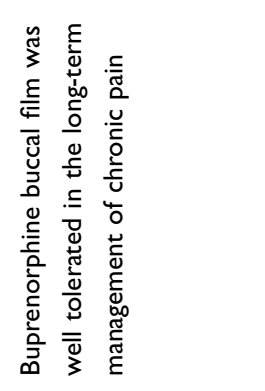 & 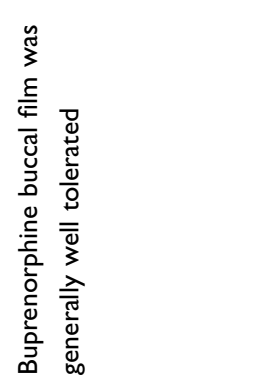 & 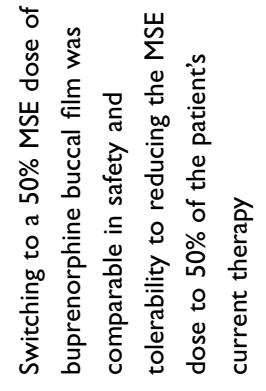 \\
\hline 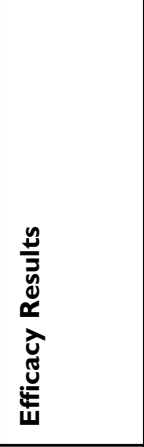 & 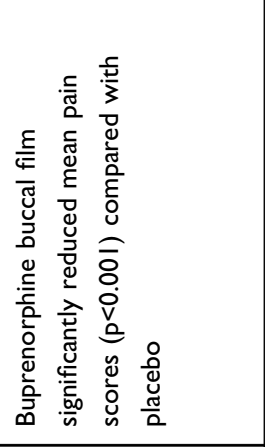 & 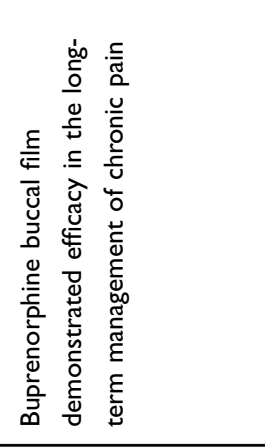 & 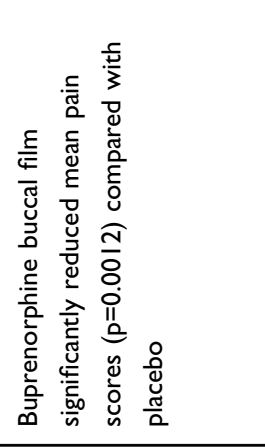 & 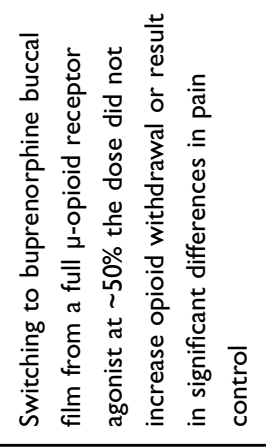 \\
\hline 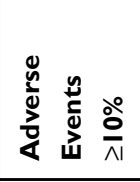 & 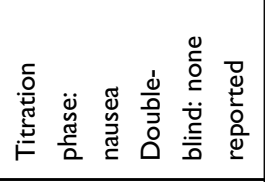 & 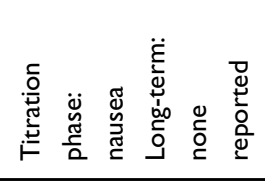 & 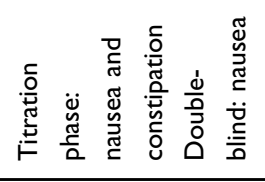 & 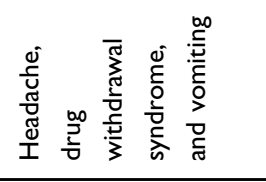 \\
\hline 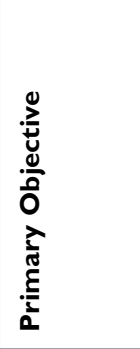 & 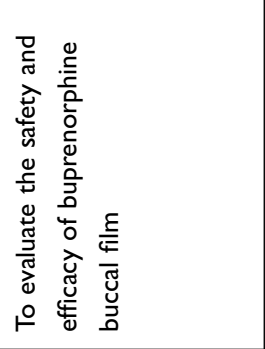 & 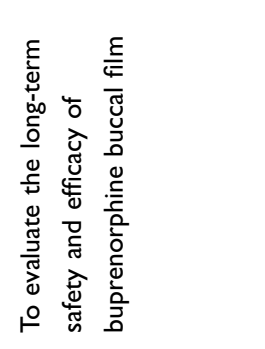 & 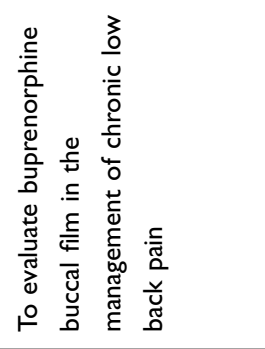 & 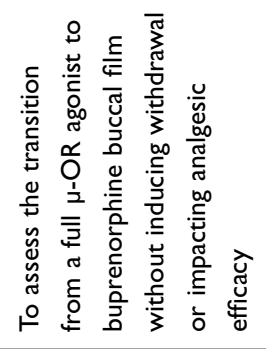 \\
\hline 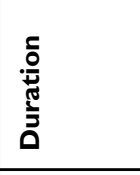 & 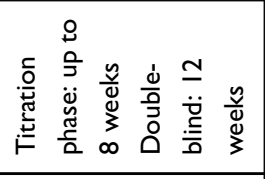 & 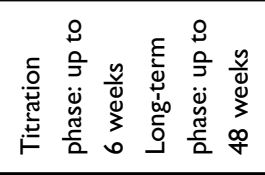 & 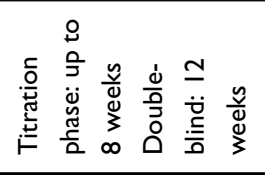 & 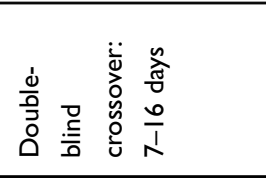 \\
\hline 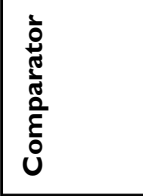 & $\begin{array}{l}\circ \\
\stackrel{\mathscr{Q}}{\mathscr{~}} \\
\frac{\pi}{0} \\
\end{array}$ & 1 & $\begin{array}{l}\circ \\
\stackrel{8}{\tilde{U}} \\
\frac{\pi}{0} \\
0\end{array}$ & 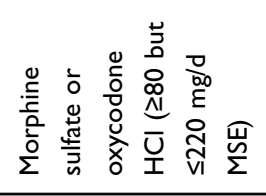 \\
\hline $\begin{array}{l}\stackrel{\Delta}{~} \\
\stackrel{\Delta}{0}\end{array}$ & 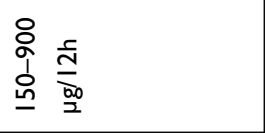 & 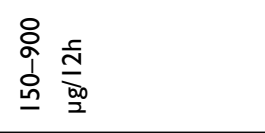 & 品 & 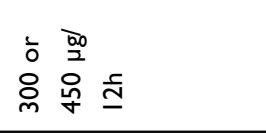 \\
\hline 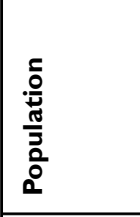 & 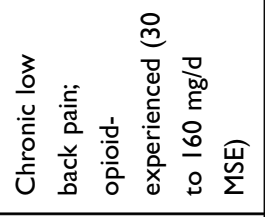 & 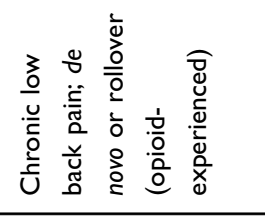 & 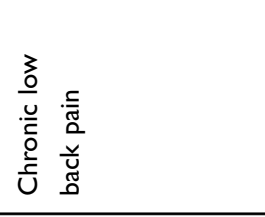 & 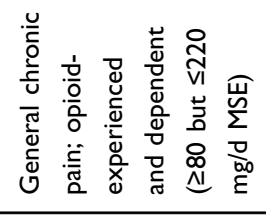 \\
\hline 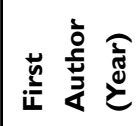 & 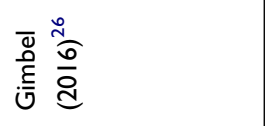 & 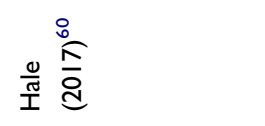 & 总 & 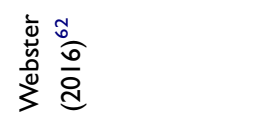 \\
\hline
\end{tabular}


support buprenorphine buccal film as an effective analgesic in patients with chronic pain.

A total of $14 \%$ of chronic pain patients discontinued transdermal buprenorphine because of lack of efficacy compared with $5 \%$ who discontinued buprenorphine buccal film for the same reason. ${ }^{23,26,60,61}$ In a responder analysis of $\geq 30 \%$ or $\geq 50 \%$ reduction in pain intensity in opioid-experienced patients, compared with transdermal buprenorphine efficacy, the efficacy of buprenorphine buccal film was more similar to that of full $\mu$-opioid receptor agonists, including hydromorphone hydrochloride ER, hydrocodone hydrochloride ER, and oxymorphone hydrochloride ER (Figure 2). ${ }^{26,53,67-70}$ However, head-to-head trials are needed to substantiate any differences in efficacy given variations in trial methodology (ie, study design, patient characteristics, use of rescue medications, and methodology used for the imputation of missing data). Nonetheless, current clinical data support the use of buprenorphine for effective chronic pain management with efficacy potentially similar to that of full $\mu$-opioid receptor agonists.

To our knowledge, there are no clinical trials of buccal buprenorphine film for acute pain, but transdermal buprenorphine has proven efficacy in the treatment of postsurgical acute pain. ${ }^{71-76}$ Future studies may provide more information regarding additional uses for buprenorphine in pain management; however, these formulations are not currently FDA approved for acute pain treatment.

\section{The Safety Of Buprenorphine In Chronic Pain}

The associated risks of abuse and addiction potential, along with the prominent adverse effects of constipation and respiratory depression, limit the use of full $\mu$-opioid receptor agonists for the management of chronic pain. ${ }^{26}$ The frequency of constipation with ER full $\mu$-opioid receptor agonists has reportedly ranged from $8 \%$ to $31 \%$, compared with $4 \%$ for buprenorphine buccal film and $13 \%$ for transdermal buprenorphine. ${ }^{28,29,70,77-82}$ In a post-marketing surveillance study, 128 (1\%) of 13,179 patients receiving transdermal buprenorphine experienced constipation. ${ }^{63}$ When constipation is a concern, buprenorphine may be a more suitable treatment than other opioids.

In clinical studies, the incidence of respiratory depression with systemic or spinal opioids ranged from $1 \%$ to $11 \% .{ }^{23}$ A post-marketing survey of 1005 patients receiving transdermal fentanyl reported respiratory depression in $8(0.8 \%)$ patients. ${ }^{83}$ Intravenous buprenorphine was shown to exhibit a ceiling effect on respiratory depression at higher doses, unlike morphine and fentanyl, which have a dose-proportional impact on respiratory depression (Figure 3) ${ }^{84,85}$ This finding is consistent with a postmarketing survey of 13,179 patients receiving treatment with transdermal buprenorphine, in which respiratory depression was reported in $1(0.01 \%)$ patient, approximately 80 times less than the incidence with transdermal fentanyl. No cases of respiratory depression have been reported in currently available buprenorphine buccal film studies. ${ }^{26,60,61}$ In addition, a panel of experts reviewing opioid pharmacology concluded that buprenorphine was the only opioid to exhibit a ceiling effect on respiratory depression. ${ }^{86}$ Buprenorphine also has a lower risk for abuse potential than Schedule II opioids, hence its Schedule III classification by the DEA. ${ }^{11,87}$ The risks of drug dependence and analgesic tolerance were also lower for buprenorphine than for Schedule II full $\mu$-opioid receptor agonists. ${ }^{87-89}$
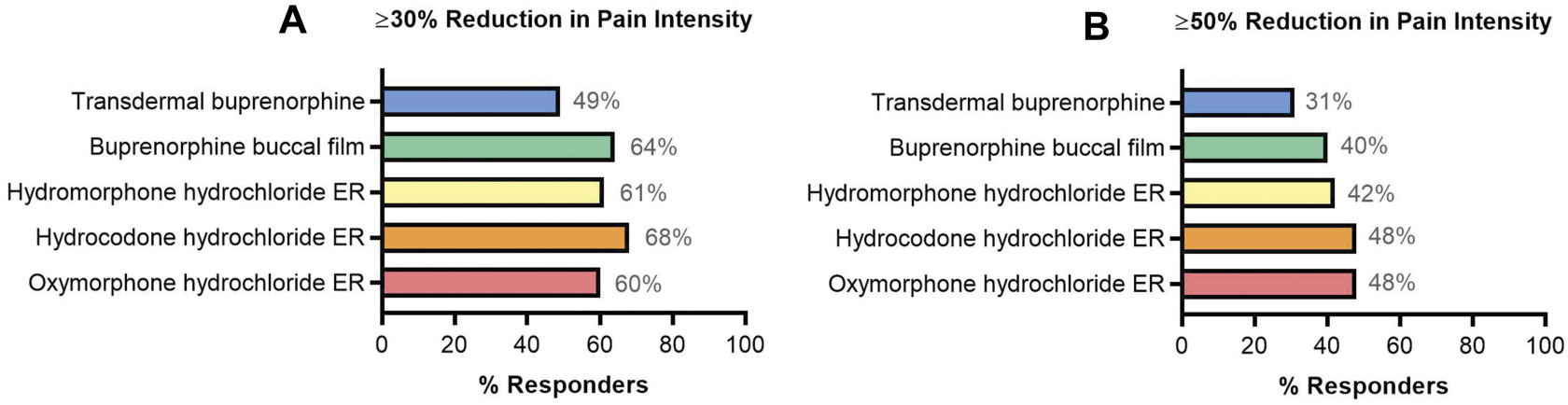

Figure 2 Responder analysis: similar trials of opioids in opioid-experienced chronic pain populations. Compared with the efficacy data for transdermal buprenorphine $(20 \mu g / h){ }^{53}$ buprenorphine buccal film ( $150-900 \mu \mathrm{g} / \mathrm{I} 2 \mathrm{~h})^{26}$ had more similar efficacy results to studies of the Schedule Il opioids hydromorphone hydrochloride ER (I2-64 mg), ${ }^{67}$ hydrocodone hydrochloride ER (20-100 mg//2h), ${ }^{69}$ and oxymorphone hydrochloride ER $(20-260 \mathrm{mg})^{70}$ assessed by $\geq 30 \%$ (A) and $\geq 50 \%$ (B) reduction in pain intensity.

Abbreviation: ER, extended-release. 


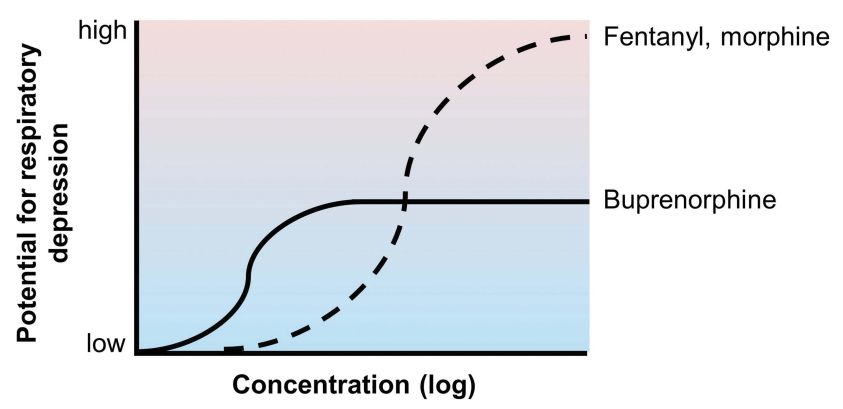

Figure 3 Conceptual representation of buprenorphine's ceiling effect on respiratory depression. Unlike the full $\mu$-opioid receptor agonists fentanyl and morphine, buprenorphine exhibits a ceiling effect on respiratory depression. ${ }^{84,85}$ The low incidence of buprenorphine-associated respiratory depression has been observed clinically. ${ }^{26,60,61,63}$

Although both transdermal buprenorphine and buprenorphine buccal film have been well tolerated in clinical studies and have additional safety benefits compared with full $\mu$ opioid receptor agonists, the buccal formulation has the additional advantage of reducing delivery site irritation compared with the transdermal patch. Application site reactions occurred in 1534 (23\%) of the 6566 patients treated with transdermal buprenorphine, ${ }^{90}$ whereas these events have not been reported with the buccal formulation. In addition, $23 \%$ of patients discontinued open-label titration with transdermal buprenorphine because of adverse events, compared with $12.5 \%$ of patients taking buprenorphine buccal film. ${ }^{26,29,61}$ When the adverse events reported in clinical trials of transdermal buprenorphine and buprenorphine buccal film were compared with those associated with ER Schedule II opioids, patients treated with buprenorphine buccal film were less likely to experience an adverse reaction in response to treatment (Figure 4). ${ }^{54,61,66}$ Buprenorphine was well tolerated in patients with chronic pain, while also exhibiting a favorable safety profile compared with full $\mu$-opioid receptor agonists, and the buccal film may confer additional safety advantages compared with the transdermal patch. However, some patients may experience adhesion issues with buprenorphine buccal film.

Regarding post-marketing experiences, the FDA Adverse Events Reporting System (FAERS) Public Dashboard received 314 adverse events reports for buprenorphine buccal film and 26,531 for transdermal buprenorphine from 2016 to March 31, 2019. A total of 73 (23.2\%) people reported drug ineffectiveness for buprenorphine buccal film vs $603(2.3 \%)$ people for transdermal

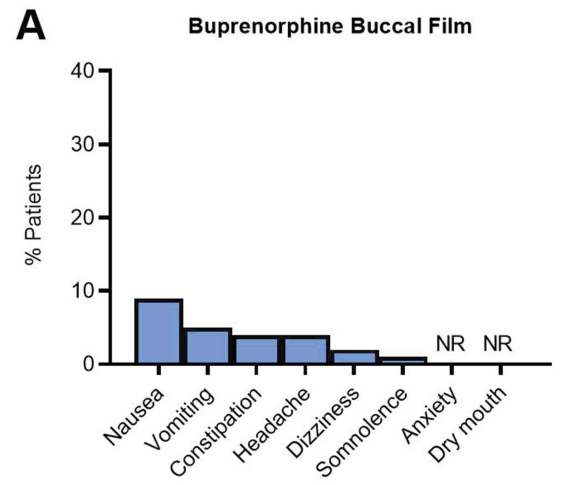

D

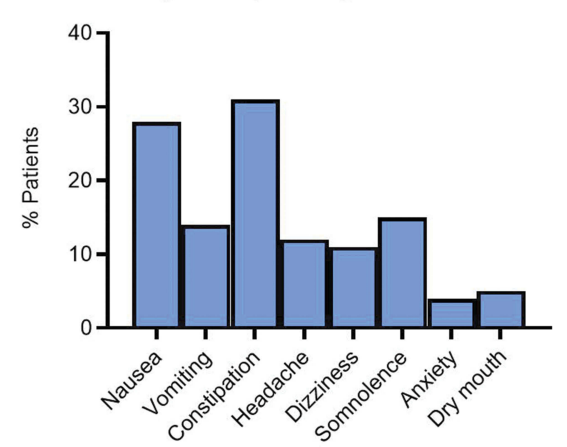

B

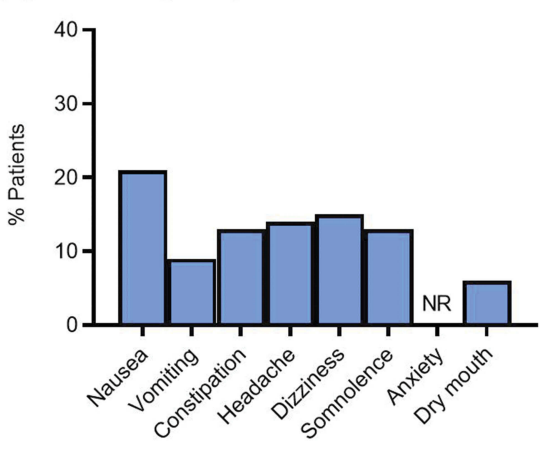

E

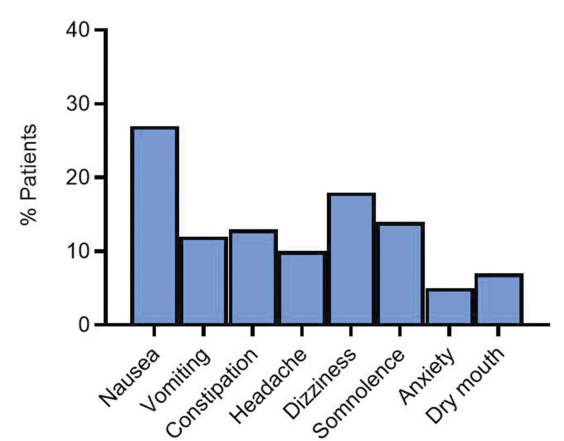

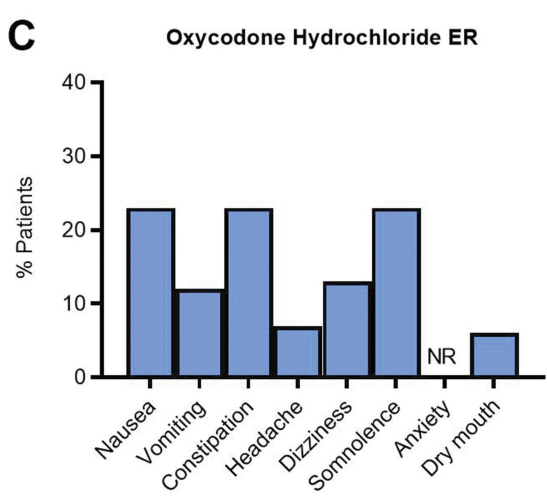

$\mathbf{F}$

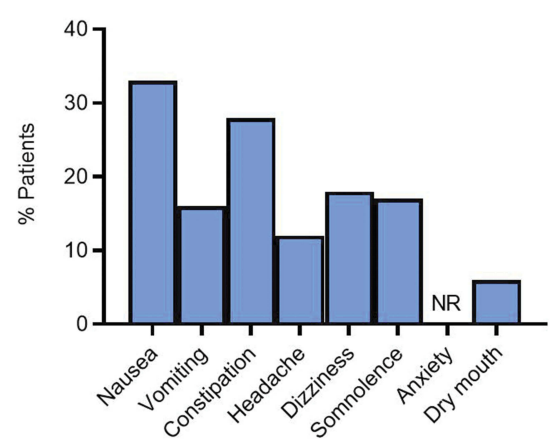

Figure 4 Safety analysis: adverse reactions reported in clinical trials of buprenorphine formulations and common Schedule II opioids for chronic pain. The percentage of patients who reported adverse reactions in clinical trials for buprenorphine buccal film $(\mathbf{A})^{28}$ is lower than those reported for the buprenorphine transdermal patch (B), ${ }^{29}$ oxycodone hydrochloride ER (C), ${ }^{79}$ hydromorphone hydrochloride ER (D), ${ }^{77}$ tapentadol ER (E), ${ }^{81}$ and oxymorphone hydrochloride ER (F). ${ }^{70}$

Abbreviations: ER, extended-release; NR, not reported. 
buprenorphine; $22(7.0 \%)$ people reported product adhesion issues for buprenorphine buccal film vs $626(2.4 \%)$ people for transdermal buprenorphine. However, this discrepancy may be due to the large variation in numbers of reports, potentially because duplicate reports are being filed in the FAERS system. In addition, data on drug exposure, concomitant medication use, titration to effect, proper use, and suspected causality are not provided in the database, nor is the total number of patients treated with a particular drug. As such, these data alone cannot be used to estimate the incidence of reactions reported or to produce an accurate comparison across drugs.

\section{The Clinical Utility Of Buprenorphine In Chronic Pain Management}

Buprenorphine is suitable for use in multiple patient populations. Buprenorphine can be used in patients with a dual diagnosis of chronic pain and opioid use disorder, those requiring concomitant medications (as fewer interactions may occur with other drugs), those with renal or hepatic impairment, and in the elderly. ${ }^{86}$ The use of buprenorphine in patients with cardiac conditions or concomitant use with antiarrhythmic agents was initially a concern, as therapeutic doses were thought to prolong the QT interval. ${ }^{23}$ Studies have since concluded that no clinically significant prolongation in the QT interval is observed within therapeutic dose ranges for various buprenorphine formulations. ${ }^{34,91-96}$ Buprenorphine also has additional benefits in that it is not immunosuppressive, ${ }^{23,97}$ does not negatively impact the hypothalamic-pituitary-adrenal pathway, ${ }^{98-100}$ and reduces anxiety and depression. ${ }^{101-103}$

Before an opioid therapy for chronic pain management is started or switched, risks and benefits should be weighed on the basis of the patient's needs. There appears to be a general improvement in the risk-benefit ratio with buprenorphine compared with full $\mu$-opioid receptor agonists, ${ }^{60}$ which may make it a favorable first-line therapy for chronic pain management when nonopioid analgesics are ineffective. ${ }^{104}$ In the United States, transdermal buprenorphine patches are available in 5-, 7.5-, 10-, 15-, and 20- $\mu \mathrm{g} /$ $\mathrm{h}$ dosages, ${ }^{23}$ and buprenorphine buccal film is available in higher strengths, including $75,150,300,450,600,750$, and $900 \mu \mathrm{g} .{ }^{24}$ In opioid-experienced patients, initiation depends on prior morphine sulfate equivalent doses. Although buprenorphine is no longer listed in the Centers for Disease Control and Prevention's morphine milligram equivalent chart because it was deemed unlikely to be associated with overdose in the same dose-dependent manner as Schedule I or Schedule II opioids, the prescribing information for transdermal buprenorphine and buprenorphine buccal film contains conversion strategies for opioid-experienced patients. ${ }^{23,24,105,106}$

\section{Conclusion}

Buprenorphine is an atypical opioid that demonstrates efficacy similar to that of Schedule II full $\mu$-opioid receptor agonists in managing chronic pain while exhibiting a favorable tolerability profile, including the reduced likelihood of abuse potential and respiratory depression. Buprenorphine has additional clinical advantages, including use in multiple patient populations, such as the elderly and those with renal or hepatic impairment, and reduced likelihood of constipation and withdrawal. Regarding the formulations indicated for chronic pain, buprenorphine buccal film has higher bioavailability, has more dose ranges, and appears to be more efficacious and tolerable than the transdermal formulation on the basis of responder and safety analyses of currently available, although limited, clinical studies. However, some patients may experience adhesion issues with the buccal film, although this was not commonly reported in clinical trials. To gain further insight into the most advantageous treatment option for chronic pain, well-controlled head-to-head trials are warranted for comparisons of buprenorphine with Schedule II opioids and of transdermal buprenorphine with buprenorphine buccal film. Nonetheless, current clinical and long-term safety data support the use of buprenorphine over full $\mu$-opioid receptor agonists for the effective and preferential treatment of chronic pain at a critical time when safer and less addictive treatments are needed.

\section{Acknowledgments}

Editorial support was provided by MedLogix Communications, LLC, in cooperation with the authors, and was sponsored by BioDelivery Sciences International, Inc. This review was sponsored by BioDelivery Sciences International, Inc.

\section{Author Contributions}

Both authors contributed to the conception and design of the manuscript and the analysis and interpretation of data, critically revised the manuscript for scientific accuracy and intellectual content, approved the final manuscript for 
publication, and agree to be accountable for all aspects of the work.

\section{Disclosure}

JVP has served as a consultant/speaker and researcher for Daiichi Sankyo, US WorldMeds, BioDelivery Sciences International, Inc, Salix, Enalare, and Neumentum. He also reports non-financial supports from BioDelivery Sciences International, Inc., during the conduct of the study and outside the submitted work. RBR was a previous employee of Johnson \& Johnson, currently has academic affiliations (Temple University, University of Arizona), consults for multiple pharmaceutical companies regarding the discovery and development of opioid and nonopioid analgesics, and is a principal investigator for two (nonopioid) analgesic drug discovery/development companies, CaRafe Drug Innovation and Neumentum. RBR also reports personal fees from BioDelivery Sciences International, Inc., NEMA Research, and partnership or stock options from CaRafe Drug Innovation, Neumentum, and Enalare outside the submitted work. The authors report no other conflicts of interest in this work.

\section{References}

1. Dahlhamer J, Lucas J, Zelaya C, et al. Prevalence of chronic pain and high-impact chronic pain among adults-United States, 2016. MMWR Morb Mortal Wkly Rep. 2018;67:1001-1007. doi:10.15 585/mmwr.mm6736a2

2. Kleiber B, Jain S, Trivedi MH. Depression and pain: implications for symptomatic presentation and pharmacological treatments. Psychiatry (Edgmont). 2005;2(5):12-18.

3. National Institute on Drug Abuse. Opioid overdose crisis; 2019. Available from: https://www.drugabuse.gov/drugs-abuse/opioids/ opioid-overdose-crisis. Accessed April 4, 2019.

4. Davis MP, Pasternak G, Behm B. Treating chronic pain: an overview of clinical studies centered on the buprenorphine option. Drugs. 2018;78(12):1211-1228. doi:10.1007/s40265-018-0953-z

5. Drug Enforcement Administration. Mid-level practitioners authorization by state. Available from: https://www.deadiversion.usdoj.gov/ drugreg/practioners/mlp by state.pdf. Accessed April 4, 2019.

6. Whitemore and Whisenant. Opioid prescribing limits across the states; 2019. Available from: https://www.pharmacytimes.com/con tributor/marilyn-bulloch-pharmd-bcps/2019/02/opioid-prescribinglimits-across-the-states. Accessed April 4, 2019.

7. Dowell D, Haegerich T, Chou R. No shortcuts to safer opioid prescribing. $N$ Engl J Med. 2019;380(24):2285-2287. doi:10.10 56/NEJMp1904190

8. Lewis JW. Ring C-bridged derivatives of thebaine and oripavine. Adv Biochem Psychopharmacol. 1974;8:123-136.

9. Khanna IK, Pillarisetti S. Buprenorphine: an attractive opioid with underutilized potential in treatment of chronic pain. J Pain Res. 2015;8:859-870. doi:10.2147/JPR.S85951

10. Campbell ND, Lovell AM. The history of the development of buprenorphine as an addiction therapeutic. Ann N Y Acad Sci. 2012;1248:124-139. doi:10.1111/j.1749-6632.2011.06352.x
11. Drug Enforcement Administration Diversion Control Division. Rescheduling of buprenorphine from Schedule V to Schedule III; 2002. Available from: https://www.deadiversion.usdoj.gov/fed regs/rules/2002/fr1007.htm. Accessed March 20, 2019.

12. Butler S. Buprenorphine: clinically useful but often misunderstood. Scand J Pain. 2013;4(3):148-152. doi:10.1016/j.sjpain.2013.05.004

13. Raffa RB, Haidery M, Huang HM, et al. The clinical analgesic efficacy of buprenorphine. J Clin Pharm Ther. 2014;39(6):577583. doi:10.1111/jcpt.12196

14. Breivik H. Buprenorphine - the ideal drug for more clinical indications for an opioid? Scand J Pain. 2013;4(3):146-147. doi:10.1016/ j.sjpain.2013.05.005

15. Zubieta J, Greenwald MK, Lombardi U, et al. Buprenorphine-induced changes in mu-opioid receptor availability in male heroin-dependent volunteers: a preliminary study. Neuropsychopharmacology. 2000;23 (3):326-334. doi:10.1016/S0893-133X(00)00110-X

16. Jasinski DR, Pevnick JS, Griffith JD. Human pharmacology and abuse potential of the analgesic buprenorphine: a potential agent for treating narcotic addiction. Arch Gen Psychiatry. 1978;35 (4):501-516. doi:10.1001/archpsyc.1978.01770280111012

17. Yassen A, Olofsen E, Romberg R, Sarton E, Danhof M, Dahan A. Mechanism-based pharmacokinetic-pharmacodynamic modeling of the antinociceptive effect of buprenorphine in healthy volunteers. Anesthesiology. 2006;104(6):1232-1242. doi:10.1097/00000542200606000-00019

18. Pergolizzi J, Aloisi AM, Dahan A, et al. Current knowledge of buprenorphine and its unique pharmacological profile. Pain Pract. 2010;10(5):428-450. doi:10.1111/j.1533-2500.2010.00378.x

19. Rothman RB, Gorelick DA, Heishman SJ, et al. An open-label study of a functional opioid kappa antagonist in the treatment of opioid dependence. J Subst Abuse Treat. 2000;18(3):277-281. doi:10.1016/S0740-5472(99)00074-4

20. Ahmadi J, Jahromi MS, Ehsaei Z. The effectiveness of different singly administered high doses of buprenorphine in reducing suicidal ideation in acutely depressed people with co-morbid opiate dependence: a randomized, double-blind, clinical trial. Trials. 2018;19(1):462. doi:10.1186/s13063-018-2843-9

21. Stein C, Machelska H. Modulation of peripheral sensory neurons by the immune system: implications for pain therapy. Pharmacol Rev. 2011;63(4):860-881. doi:10.1124/pr.110.003145

22. Likar R. Transdermal buprenorphine in the management of persistent pain: safety aspects. Ther Clin Risk Manag. 2006;2(1):115-125.

23. Davis MP. Twelve reasons for considering buprenorphine as a frontline analgesic in the management of pain. J Support Oncol. 2012;10(6):209-219. doi:10.1016/j.suponc.2012.05.002

24. United States Health and Human Services. HHS acting secretary declares public health emergency to address national opioid crisis. Available from: https://www.hhs.gov/about/news/2017/10/26/hhsacting-secretary-declares-public-health-emergency-addressnational-opioid-crisis.html. Accessed January 24, 2019.

25. United States Department of Health and Human Services. Pain management best practices inter-agency task force report: updates, gaps, inconsistencies, and recommendations; 2019. Available from: https://www.hhs.gov/ash/advisory-committees/pain/reports/index. html. Accessed September 17, 2019.

26. Gimbel J, Spierings EL, Katz N, Xiang Q, Tzanis E, Finn A. Efficacy and tolerability of buccal buprenorphine in opioid-experienced patients with moderate to severe chronic low back pain: results of a phase 3, enriched enrollment, randomized withdrawal study. Pain. 2016;157(11):2517-2526. doi:10.1097/j.pain.000000 0000000670

27. Kuhlman JJ Jr., Lalani S, Magluilo J Jr., Levine B, Darwin WD. Human pharmacokinetics of intravenous, sublingual, and buccal buprenorphine. J Anal Toxicol. 1996;20(6):369-378. doi:10.1093/ jat $/ 20.6 .369$ 
28. Belbuca ${ }^{\circledR}$ (buprenorphine buccal film) [prescribing information]. Raleigh, NC: BioDelivery Sciences International, Inc.; 2018.

29. Butrans ${ }^{\circledR}$ (buprenorphine transdermal system) [prescribing information]. Stamford, CT: Purdue Pharma L.P.; 2018.

30. Mendelson J, Upton RA, Everhart ET, Jacob P 3rd, Jones RT. Bioavailability of sublingual buprenorphine. J Clin Pharmacol. 1997;37(1):31-37. doi:10.1177/009127009703700106

31. Böhme K. Buprenorphine in a transdermal therapeutic system: a new option. Clin Rheumatol. 2002;21(1 Suppl.):S13-S16. doi:10.1007/s100670200031

32. Breivik H, Ljosaa TM, Stengaard-Pedersen K, et al. A 6-months, randomised, placebo-controlled evaluation of efficacy and tolerability of a low-dose 7-day buprenorphine transdermal patch in osteoarthritis patients naïve to potent opioids. Scand J Pain. 2010;1(3):122-141. doi:10.1016/j.sjpain.2010.05.035

33. Gianni W, Madaio AR, Ceci M, et al. Transdermal buprenorphine for the treatment of chronic noncancer pain in the oldest old. J Pain Symptom Manage. 2011;41(4):707-714. doi:10.1016/j.jpainsymman. 2010.06.015

34. Gordon A, Callaghan D, Spink D, et al. Buprenorphine transdermal system in adults with chronic low back pain: a randomized, doubleblind, placebo-controlled crossover study, followed by an openlabel extension phase. Clin Ther. 2010;32(5):844-860. doi:10.1016/j.clinthera.2010.04.018

35. Gordon A, Rashiq S, Moulin DE, et al. Buprenorphine transdermal system for opioid therapy in patients with chronic low back pain. Pain Res Manage. 2010;15(3):169-178. doi:10.1155/2010/ 216725

36. Hakl M. Transdermal buprenorphine in clinical practice: a multicenter, postmarketing study in the Czech Republic, with a focus on neuropathic pain components. Pain Manage. 2012;2(2):169-175. doi: $10.2217 /$ pmt. 11.92

37. Karlsson J, Söderström A, Augustini BG, Berggren AC. Is buprenorphine transdermal patch equally safe and effective in younger and elderly patients with osteoarthritis-related pain? Results of an age-group controlled study. Curr Med Res Opin. 2014;30(4):575587. doi:10.1185/03007995.2013.873714

38. Karlsson M, Berggren AC. Efficacy and safety of low-dose transdermal buprenorphine patches $(5,10$, and $20 \mu \mathrm{g} / \mathrm{h})$ versus prolonged-release tramadol tablets $(75,100,150$, and $200 \mathrm{mg})$ in patients with chronic osteoarthritis pain: a 12-week, randomized, open-label, controlled, parallel-group noninferiority study. Clin Ther. 2009;31(3):503-513. doi:10.1016/j.clinthera. 2009.03.001

39. Leng X, Li Z, Lv H, et al. Effectiveness and safety of transdermal buprenorphine versus sustained-release tramadol in patients with moderate to severe musculoskeletal pain: an 8-week, randomized, double-blind, double-dummy, multicenter, active-controlled, noninferiority study. Clin J Pain. 2015;31(7):612-620. doi:10.1097/ AJP.0000000000000144

40. Likar R, Kayser H, Sittl R. Long-term management of chronic pain with transdermal buprenorphine: a multicenter, open-label, followup study in patients from three short-term clinical trials. Clin Ther. 2006;28(6):943-952. doi:10.1016/j.clinthera.2006.06.012

41. Likar R, Lorenz V, Korak-Leiter M, Kager I, Sittl R. Transdermal buprenorphine patches applied in a 4-day regimen versus a 3-day regimen: a single-site, phase III, randomized, open-label, crossover comparison. Clin Ther. 2007;29(8):1591-1606. doi:10.1016/j. clinthera.2007.08.001

42. Likar R, Vadlau EM, Breschan C, Kager I, Korak-Leiter M, Ziervogel G. Comparable analgesic efficacy of transdermal buprenorphine in patients over and under 65 years of age. Clin J Pain. 2008;24(6):536-543. doi:10.1097/AJP.0b013e31816 $73 \mathrm{~b} 65$
43. Miller K, Yarlas A, Wen W, et al. Buprenorphine transdermal system and quality of life in opioid-experienced patients with chronic low back pain. Expert Opin Pharmacother. 2013;14 (3):269-277. doi:10.1517/14656566.2013.767331

44. Miller K, Yarlas A, Wen W, et al. The impact of buprenorphine transdermal delivery system on activities of daily living among patients with chronic low back pain: an application of the international classification of functioning, disability and health. Clin J Pain. 2014;30(12):1015-1022. doi:10.1097/AJP.0000000000000068

45. Pace MC, Passavanti MB, Grella E, et al. Buprenorphine in longterm control of chronic pain in cancer patients. Front Biosci. 2007;12(4):1291-1299. doi:10.2741/2147

46. Pota V, Barbarisi M, Sansone P, et al. Combination therapy with transdermal buprenorphine and pregabalin for chronic low back pain. Pain Manage. 2012;2(1):23-31. doi:10.2217/pmt.11.71

47. Ripa SR, McCarberg BH, Munera C, Wen W, Landau CJ. A randomized, 14-day, double-blind study evaluating conversion from hydrocodone/acetaminophen (Vicodin) to buprenorphine transdermal system $10 \mu \mathrm{g} / \mathrm{h}$ or $20 \mu \mathrm{g} / \mathrm{h}$ in patients with osteoarthritis pain. Expert Opin Pharmacother. 2012;13(9):1229-1241. doi:10.1517/14656566.2012.667073

48. Serpell M, Tripathi S, Scherzinger S, Rojas-Farreras S, Oksche A, Wilson M. Assessment of transdermal buprenorphine patches for the treatment of chronic pain in a UK observational study. Patient. 2016;9(1):35-46. doi:10.1007/s40271-015-0151-y

49. Silverman S, Raffa RB, Cataldo MJ, Kwarcinski M, Ripa SR. Use of immediate-release opioids as supplemental analgesia during management of moderate-to-severe chronic pain with buprenorphine transdermal system. J Pain Res. 2017;10:1255-1263. doi: $10.2147 / J P R$

50. Sittl R, Griessinger N, Likar R. Analgesic efficacy and tolerability of transdermal buprenorphine in patients with inadequately controlled chronic pain related to cancer and other disorders: a multicenter, randomized, double-blind, placebo-controlled trial. Clin Ther. 2003;25(1):150-168. doi:10.1016/S0149-2918(03)90019-1

51. Skvarc NK. Transdermal buprenorphine in clinical practice: results from a multicenter, noninterventional postmarketing study in Slovenia. Pain Manag. 2012;2(2):177-183. doi:10.2217/pmt.11.91

52. Sorge J, Sittl R. Transdermal buprenorphine in the treatment of chronic pain: results of a phase III, multicenter, randomized, double-blind, placebo-controlled study. Clin Ther. 2004;26(11):18081820. doi:10.1016/j.clinthera.2004.11.008

53. Steiner D, Munera C, Hale M, Ripa S, Landau C. Efficacy and safety of buprenorphine transdermal system (BTDS) for chronic moderate to severe low back pain: a randomized, double-blind study. J Pain. 2011;12(11):1163-1173. doi:10.1016/j.jpain.2011. 06.003

54. Steiner DJ, Sitar S, Wen W, et al. Efficacy and safety of the seven-day buprenorphine transdermal system in opioid-naïve patients with moderate to severe chronic low back pain: a enriched, randomized, doubleblind, placebo-controlled study. J Pain Symptom Manage. 2011;42 (6):903-917. doi:10.1016/j.jpainsymman.2011.04.006

55. Vondráčková D. Transdermal buprenorphine in clinical practice: a multicenter, noninterventional postmarketing study in the Czech Republic. Pain Manage. 2012;2(2):163-168. doi:10.2217/pmt.11.93

56. Yarlas A, Miller K, Wen W, et al. A randomized, placebo-controlled study of the impact of the 7-day buprenorphine transdermal system on health-related quality of life in opioid-naïve patients with moderate-to-severe chronic low back pain. J Pain. 2013;14 (1):14-23. doi:10.1016/j.jpain.2012.09.016

57. Yarlas A, Miller K, Wen W, et al. Buprenorphine transdermal system compared with placebo reduces interference in functioning for chronic low back pain. Postgrad Med. 2015;127(1):38-45. doi:10.1080/00325481.2014.992715 
58. James IG, O'Brien CM, McDonald CJ. A randomized, doubleblind, double-dummy comparison of the efficacy and tolerability of low-dose transdermal buprenorphine (BuTrans seven-day patches) with buprenorphine sublingual tablets (Temgesic) in patients with osteoarthritis pain. J Pain Symptom Manage. 2010;40(2):266-278. doi:10.1016/j.jpainsymman.2010.01.013

59. Yarlas A, Miller K, Wen W, et al. Buprenorphine transdermal system improves sleep quality and reduces sleep disturbance in patients with moderate-to-severe chronic low back pain: results from two randomized controlled trials. Pain Pract. 2016;16 (3):345-358. doi:10.1111/papr.2016.16.issue-3

60. Hale M, Urdaneta V, Kirby MT, Xiang Q, Rauck R. Long-term safety and analgesic efficacy of buprenorphine buccal film in patients with moderate-to-severe chronic pain requiring aroundthe-clock opioids. J Pain Res. 2017;10:233-240. doi:10.2147/JPR

61. Rauck RL, Potts J, Xiang Q, Tzanis E, Finn A. Efficacy and tolerability of buccal buprenorphine in opioid-naive patients with moderate to severe chronic low back pain. Postgrad Med. 2016;128 (1):1-11. doi:10.1080/00325481.2016.1128307

62. Webster L, Gruener D, Kirby T, Xiang Q, Tzanis E, Finn A. Evaluation of the tolerability of switching patients on chronic full $\mu$-opioid agonist therapy to buccal buprenorphine. Pain Med. 2016;17(5):899-907.

63. Griessinger N, Sittl R, Likar R. Transdermal buprenorphine in clinical practice: a post-marketing surveillance study in 13,179 patients. Curr Med Res Opin. 2005;21(8):1147-1156. doi:10.1185/030079905X53315

64. Corli O, Floriani I, Roberto A, et al. Are strong opioids equally effective and safe in the treatment of chronic cancer pain? A multicenter randomized phase IV 'real life' trial on the variability of response to opioids. Ann Oncol. 2016;27(6):1107-1115. doi:10.1093/annonc/mdw097

65. Wolff RF, Aune D, Truyers C, et al. Systematic review of efficacy and safety of buprenorphine versus fentanyl or morphine in patients with chronic moderate to severe pain. Curr Med Res Opin. 2012;28 (5):833-845. doi:10.1185/03007995.2012.678938

66. Katz N, Rauck R, Ahdieh H, et al. A 12-week, randomized, placebo-controlled trial assessing the safety and efficacy of oxymorphone extended release for opioid-naive patients with chronic low back pain. Curr Med Res Opin. 2007;23(1):117-128. doi:10.1185/030079906X162692

67. Hale M, Khan A, Kutch M, Li S. Once-daily OROS hydromorphone ER compared with placebo in opioid-tolerant patients with chronic low back pain. Curr Med Res Opin. 2010;26(6):15051518. doi:10.1185/03007995.2010.484723

68. Hale ME, Ahdieh H, Ma T, Rauck R; Oxymorphone ER Study Group. Efficacy and safety of OPANA ER (oxymorphone extended release) for relief of moderate to severe chronic low back pain in opioid-experienced patients: a 12-week, randomized, double-blind, placebo-controlled study. J Pain. 2007;8(2):175-184. doi:10.1016/j.jpain.2006.09.011

69. Rauck RL, Nalamachu S, Wild JE, et al. Single-entity hydrocodone extended-release capsules in opioid-tolerant subjects with moderate-to-severe chronic low back pain: a randomized double-blind, placebo-controlled study. Pain Med. 2014;15(6):975-985. doi:10. $1111 /$ pme. 12377

70. Opana ${ }^{\circledR}$ ER (oxymorphone $\mathrm{HCl}$ extended-release tablets) [prescribing information]. Malvern, PA: Endo Pharmaceuticals, Inc; 2018.

71. Arshad Z, Prakash R, Gautam S, Kumar S. Comparison between transdermal buprenorphine and transdermal fentanyl for postoperative pain relief after major abdominal surgeries. J Clin Diagn Res. 2015;9(12):UC01-04. doi:10.7860/JCDR/2015/16327.6917

72. Desai SN, Badiger SV, Tokur SB, Naik PA. Safety and efficacy of transdermal buprenorphine versus oral tramadol for the treatment of post-operative pain following surgery for fracture neck of femur: a prospective, randomised clinical study. Indian J Anaesth. 2017;61 (3):225-229. doi:10.4103/ija.IJA_208_16
73. Kim HJ, Ahn HS, Nam Y, Chang BS, Lee CK, Yeom JS. Comparative study of the efficacy of transdermal buprenorphine patches and prolonged-release tramadol tablets for postoperative pain control after spinal fusion surgery: a prospective, randomized controlled non-inferiority trial. Eur Spine J. 2017;26(11):29612968. doi: $10.1007 / \mathrm{s} 00586-017-5213-5$

74. Kumar S, Chaudhary AK, Singh PK, et al. Transdermal buprenorphine patches for postoperative pain control in abdominal surgery. J Clin Diagn Res. 2016;10(6):UC05-08.

75. Lee JH, Kim JH, Kim JH, et al. Efficacy and safety of transdermal buprenorphine versus oral tramadol/acetaminophen in patients with persistent postoperative pain after spinal surgery. Pain Res Manag. 2017;2017:2071494. doi:10.1155/2017/2071494

76. Xu C, Li M, Wang C, Li H, Liu H. Perioperative analgesia with a buprenorphine transdermal patch for hallux valgus surgery: a prospective, randomized, controlled study. J Pain Res. 2018;11:867873. doi: $10.2147 / \mathrm{JPR}$

77. Exalgo ${ }^{\circledR}$ (hydromorphone $\mathrm{HCl}$ extended-release tablets) [prescribing information]. Hazelwood, MO: Mallinckrodt; 2016.

78. Duragesic ${ }^{\circledR}$ (fentanyl transdermal system) [prescribing information]. Titusville, NJ: Janssen Pharmaceuticals, Inc; 2016.

79. Oxycontin ${ }^{\circledR}$ (oxycodone $\mathrm{HCl}$ extended-release tablets) [prescribing information]. Stamford, CT: Purdue Pharma L.P.; 2018.

80. Zohydro ${ }^{\circledR}$ ER (hydrocodone bitartrate extended-release capsules) [prescribing information]. Morristown, NJ: Pernix Therapeutics, LLC.; 2016.

81. Nucynta ${ }^{\circledR}$ ER (tapentadol extended-release tablets) [prescribing information]. Newark, CA: Depomed, Inc; 2018.

82. Kadian ${ }^{\circledR}$ (morphine sulfate extended-release capsules) [prescribing information]. Madison, NJ: Allergan USA, Inc.; 2018.

83. Radbruch L, Sabatowski R, Petzke F, Brunsch-Radbruch A, Grond S, Lehmann KA. Transdermal fentanyl for the management of cancer pain: a survey of 1005 patients. Palliat Med. 2001;15 (4):309-321. doi:10.1191/026921601678320296

84. Dahan A, Yassen A, Bijl H, et al. Comparison of the respiratory effects of intravenous buprenorphine and fentanyl in humans and rats. Br J Anaesth. 2005;94(6):825-834. doi:10.1093/bja/aei145

85. Dahan A, Yassen A, Romberg R, et al. Buprenorphine induces ceiling in respiratory depression but not in analgesia. $\mathrm{Br} J$ Anaesth. 2006;96(5):627-632. doi:10.1093/bja/ael051

86. Pergolizzi J, Boger RH, Budd K, et al. Opioids and the management of chronic severe pain in the elderly: consensus statement of an international expert panel with focus on the six clinically most often used World Health Organization Step III opioids (buprenorphine, fentanyl, hydromorphone, methadone, morphine, oxycodone). Pain Pract. 2008;8(4):287-313. doi:10.1111/j.1533-2500. 2008.00204.x

87. Walsh SL, Eissenberg T. The clinical pharmacology of buprenorphine: extrapolating from the laboratory to the clinic. Drug Alcohol Depend. 2003;70(2 Supp1.):S13-S27. doi:10.1016/S0376-8716(03) 00056-5

88. Robinson SE. Buprenorphine: an analgesic with an expanding role in the treatment of opioid addiction. CNS Drug Rev. 2002;8 (4):377-390. doi:10.1111/j.1527-3458.2002.tb00235.x

89. Walsh SL, Preston KL, Bigelow GE, Stitzer ML. Acute administration of buprenorphine in humans: partial agonist and blockade effects. J Pharmacol Exp Ther. 1995;274(1):361-372.

90. Wen W, Lynch SY, Munera C, Swanton R, Ripa SR, Maibach H. Application site adverse events associated with the buprenorphine transdermal system: a pooled analysis. Expert Opin Drug Saf. 2013;12(3):309-319. doi:10.1517/14740338.2013.780025

91. Schmith VD, Curd L, Lohmer LRL, Laffont CM, Andorn A, Young MA. Evaluation of the effects of a monthly buprenorphine depot subcutaneous injection on QT interval during treatment for opioid use disorder. Clin Pharmacol Ther. 2019;106(3):576-584. doi:10. $1002 /$ cpt.1406 
92. de Jong IM, de Ruiter GS. Buprenorphine as a safe alternative to methadone in a patient with acquired long QT syndrome: a case report Neth Heart J. 2013;21(5):249-252. doi:10.1007/s12471-011-0073-9

93. Kao DP, Haigney MC, Mehler PS, Krantz MJ. Arrhythmia associated with buprenorphine and methadone reported to the food and drug administration. Addiction. 2015;110(9):1468-1475. doi:10.11 11/add.13013

94. Poole SA, Pecoraro A, Subramaniam G, Woody G, Vetter VL. Presence or absence of QTc prolongation in buprenorphine-naloxone among youth with opioid dependence. J Addict Med. 2016;10 (1):26-33. doi:10.1097/ADM.0000000000000176

95. Stallvik M, Nordstrand B, Kristensen O, Bathen J, Skogvoll E, Spigset O. Corrected QT interval during treatment with methadone and buprenorphine: relation to doses and serum concentrations Drug Alcohol Depend. 2013;129(1-2):88-93. doi:10.1016/j. drugalcdep.2012.09.016

96. Darpo B, Zhou M, Bai SA, Ferber G, Xiang Q, Finn A. Differentiating the effect of an opioid agonist on cardiac repolarization from micro-receptor-mediated, indirect effects on the QT interval: a randomized, 3-way crossover study in healthy subjects. Clin Ther. 2016;38(2):315-326. doi:10.1016/j.clinthera.2015.12. 004

97. Budd K. Pain management: is opioid immunosuppression a clinical problem? Biomed Pharmacother. 2006;60(7):310-317. doi:10.10 16/j.biopha.2006.06.015

98. Hallinan R, Byrne A, Agho K, McMahon CG, Tynan P, Attia J. Hypogonadism in men receiving methadone and buprenorphine maintenance treatment. Int $J$ Androl. 2009;32(2):131-139. doi:10.1111/ija.2009.32.issue-2
99. Hallinan R, Byrne A, Agho K, McMahon C, Tynan P, Attia J. Erectile dysfunction in men receiving methadone and buprenorphine maintenance treatment. J Sex Med. 2008;5(3):684-692. doi:10.1111/j.1743-6109.2007.00702.x

100. Aurilio C, Ceccarelli I, Pota V, et al. Endocrine and behavioural effects of transdermal buprenorphine in pain-suffering women of different reproductive ages. Endocr J. 2011;58(12):1071-1078. doi:10.1507/endocrj.EJ11-0095

101. Karp JF, Butters MA, Begley AE, et al. Safety, tolerability, and clinical effect of low-dose buprenorphine for treatment-resistant depression in midlife and older adults. J Clin Psychiatry. 2014;75 (8):e785-e793. doi:10.4088/JCP.13m08725

102. Bodkin JA, Zornberg GL, Lukas SE, Cole JO. Buprenorphine treatment of refractory depression. J Clin Psychopharmacol. 1995;15(1):49-57. doi:10.1097/00004714-199502000-00008

103. Ahmadi J, Jahromi MS. Anxiety treatment of opioid dependent patients with buprenorphine: a randomized, double-blind, clinical trial. Indian J Psychol Med. 2017;39(4):445-449. doi:10.4103/ 0253-7176.211765

104. Ballantyne JC. Opioid therapy in chronic pain. Phys Med Rehabil Clin N Am. 2015;26(2):201-218. doi:10.1016/j.pmr.2014.12.001

105. Centers for Disease Control and Prevention. CDC guideline for prescribing opioids for chronic pain. [Updated April 17, 2019]. Available from: https://www.cdc.gov/drugoverdose/prescribing/ guideline.html. Accessed June 10, 2019.

106. American Academy of Family Physicians. CDC clarifies opioid guideline dosage thresholds. January 12, 2018. Available from: https://www.aafp.org/news/health-of-the-public/20180112cdco pioidclarify.html. Accessed June 10, 2019.
Journal of Pain Research

\section{Publish your work in this journal}

The Journal of Pain Research is an international, peer reviewed, open access, online journal that welcomes laboratory and clinical findings in the fields of pain research and the prevention and management of pain. Original research, reviews, symposium reports, hypothesis formation and commentaries are all considered for publication. The manuscript management system is completely online and includes a very quick and fair peer-review system, which is all easy to use. Visit http:// www.dovepress.com/testimonials.php to read real quotes from published authors. 